Accepted by the ApJ for the 1 Feb. 2005 issue

\title{
A RIGOROUS ATTEMPT TO VERIFY INTERSTELLAR GLYCINE
}

\author{
L. E. Snyder ${ }^{1}$, F. J. Lovas $^{2}$, J. M. Hollis ${ }^{3}$, D. N. Friedel ${ }^{1}$, P. R. Jewell ${ }^{4}$, A. Remijan ${ }^{1,3,5}$, V. \\ V. Ilyushin ${ }^{6}$, E. A. Alekseev ${ }^{6}$, and S. F. Dyubko ${ }^{6}$
}

\begin{abstract}
In 2003, Kuan, Charnley, and co-workers reported the detection of interstellar glycine $\left(\mathrm{NH}_{2} \mathrm{CH}_{2} \mathrm{COOH}\right)$ based on observations of 27 lines in 19 different spectral bands in one or more of the sources Sgr B2(N-LMH), Orion KL, and W51 e1/e2. They supported their detection report with rotational temperature diagrams for all three sources. In this paper, we present essential criteria which can be used in a straightforward analysis technique to confirm the identity of an interstellar asymmetric rotor such as glycine. We use new laboratory measurements of glycine as a basis for applying this analysis technique, both to our previously unpublished $12 \mathrm{~m}$ telescope data and to the previously published SEST data of Nummelin and colleagues. We conclude that key lines necessary for an interstellar glycine identification have not yet been found. We identify some common molecular candidates that should be examined further as more likely carriers of several of the lines reported as glycine. Finally, we illustrate that a rotational
\end{abstract}

\footnotetext{
${ }^{1}$ Department of Astronomy, University of Illinois, Urbana, IL 61801

email: snyder@astro.uiuc.edu, friedel@astro.uiuc.edu, aremijan@pop900.gsfc.nasa.gov

${ }^{2}$ Optical Technology Division, National Institute of Standards and Technology, Gaithersburg, MD 208998441

email: lovas@nist.gov

${ }^{3}$ Earth and Space Data Computing Div., Code 930, NASA's Goddard Space Flight Center, Greenbelt, MD 20771

email: Jan.M.Hollis@gsfc.nasa.gov

${ }^{4}$ National Radio Astronomy Observatory, Green Bank, WV 24944-0002

email: pjewell@nrao.edu

${ }^{5}$ National Research Council Resident Research Associate

${ }^{6}$ Institute of Radio Astronomy of NASU, Krasnoznamennaya 4, 61002 Kharkow, Ukraine

email: ilyushin@rian.ira.kharkov.ua; alekseev@rian.ira.kharkov.ua
} 
temperature diagram used without the support of correct spectroscopic assignments is not a reliable tool for the identification of interstellar molecules.

Subject headings: ISM: abundances - ISM: clouds - ISM: individual (Sagittarius B2[N-LMH], Orion KL, W51 e1/e2) - ISM: molecules - radio lines: ISM

\section{INTRODUCTION}

Interstellar glycine $\left(\mathrm{NH}_{2} \mathrm{CH}_{2} \mathrm{COOH}\right)$ has been unsuccessfully sought using both singleelement telescopes (Brown et al. 1979; Hollis et al. 1980; Snyder et al. 1983; Beralis et al. 1985; Guelin \& Cernicharo 1989; Combes, Rieu, \& Wlodarczak 1996; Ceccarelli et al. 2000) and interferometric arrays (Snyder 1997; Hollis et al. 2003a,b). Recently, Kuan et al. (2003) reported the identification of interstellar glycine $\left(\mathrm{NH}_{2} \mathrm{CH}_{2} \mathrm{COOH}\right)$ in the direction of the hot molecular cores Sgr B2(N-LMH), Orion KL, and W51 e1/e2.

We note several unusual aspects about the Kuan et al. (2003) reported glycine results. First, their glycine report was based on the detection of 27 spectral line features in the sources Orion KL, W51 e1/e2, and Sgr B2(N-LMH). They used the NRAO ${ }^{7} 12 \mathrm{~m}$ radio telescope operating in the range of 130 to $242 \mathrm{GHz}$. The 27 independent spectral line features detected in 3 different sources might seem to suggest reasonable evidence. Certainly the detection of the same ensemble of spectral lines in multiple sources is a characteristic of interstellar molecules with structural components similar to glycine, such as formic acid (HCOOH) and acetic acid $\left(\mathrm{CH}_{3} \mathrm{COOH}\right)$ (see, for example, Liu, Mehringer, \& Snyder 2001; Remijan et al. 2002). However, each of the three glycine sources was observed to contain no more than 13 to 16 of the 27 independent spectral lines features and only 3 of the 27 spectral line features were found to be common to all 3 sources. Second, 16 of the 27 reported glycine features are not the four-fold degenerate rotational transitions that should be the most likely to be detected for R-branch asymmetric rotor transitions. Third, the glycine rest frequencies used in their observations were based on a Hamiltonian calculation which was extrapolated over $100 \mathrm{GHz}$. This method can yield potentially large uncertainties in the frequency predictions. Fourth, the glycine column densities derived from rotational temperature diagrams were surprisingly high. In fact, Orion KL was reported to have the highest glycine column density, but the interstellar molecule with structure closest to glycine, acetic acid, has never been detected there (Remijan et al. 2003).

\footnotetext{
${ }^{7}$ The National Radio Astronomy Observatory (NRAO) is a facility of the National Science Foundation operated under cooperative agreement by Associated Universities, Inc.
} 
In this paper, we investigate the above concerns by starting from new laboratory measurements of glycine that improve the Hamiltonian fit and thereby result in highly reliable interpolated frequency predictions which provide the foundation for our discussion. We list and explain rigorous and essential criteria for identifying a new interstellar molecule such as glycine. Then we use these criteria on key glycine spectral lines reported by Kuan et al. (2003) to interpret independent observational data for Orion KL, W51 e1/e2, and Sgr B2(N-LMH), and show that their key lines fail the tests required for a correct glycine identification. In investigating alternative identifications, we find that the carriers of several important spectral lines which have been assigned to glycine are more logically assigned to other, more common species. Finally, we find that random choices of unidentified spectral lines can be used to generate a reasonable glycine rotational temperature diagram similar to those published by Kuan et al. (2003).

\section{GLYCINE SPECTRAL LINE ANALYSIS AND PREDICTED REST FREQUENCIES}

The microwave spectrum of conformer I of glycine was first recorded by Suenram \& Lovas (1980) from $82 \mathrm{GHz}$ to $113 \mathrm{GHz}$ in a heated parallel plate Stark cell. This study provided the rotational analysis and rough dipole moment determination. Further measurements of the ${ }^{14} \mathrm{~N}$ quadrupole coupling hyperfine structure and Stark effect on low J transitions between $16 \mathrm{GHz}$ and $24.5 \mathrm{GHz}$ were subsequently reported by Lovas et al. (1995). While these literature data provided a firm basis for predicting transitions up to about $150 \mathrm{GHz}$ for guiding past interstellar searches, the $2 \sigma$ (standard deviation) uncertainties ${ }^{8}$ ranged from $1.5 \mathrm{MHz}$ at $206 \mathrm{GHz}$ to $2.8 \mathrm{MHz}$ at $240 \mathrm{GHz}$ for the main interstellar transitions reported by Kuan et al. (2003). Recently, at Kharkow a heated quartz absorption cell was used to provide new measurements between $75 \mathrm{GHz}$ and $260 \mathrm{GHz}$ utilizing an automated synthesizer-based spectrometer described by Ilyushin et al. (2001). New glycine search frequencies could then be predicted by incorporating both the previously reported transitions and the new measurements into a rotational analysis which employed the Watson A-reduction Hamiltonian with quartic centrifugal distortion terms included (Watson 1977). The details of these laboratory measurements and Hamiltonian calculations will be reported in a future paper. For this paper, it is important to note that for the majority of the calculated transitions the uncertainties have been reduced by a factor of 10 or more from those used by Kuan et al. (2003). Thus, we are starting our analysis with highly accurate glycine rest frequency predictions.

\footnotetext{
${ }^{8}$ Unless otherwise noted, all uncertainties quoted in this paper are $2 \sigma$ or coverage factor $\mathrm{k}=2$ as described by Taylor \& Kuyatt (1994).
} 
Table 1 summarizes the reported glycine line detections in the direction of the hot molecular cores Sgr B2(N-LMH), Orion KL, and W51 e1/e2. The first column lists a number that Kuan et al. (2003) introduced to represent a particular glycine line. For the convenience of the reader, we adopt this shorthand notation hereafter referred to as the "glycine line number". The second column gives the rotational quantum numbers of the associated transitions, and the third identifies each reported transition as an a-type or a b-type. The fourth column lists our newly calculated glycine rest frequencies, and the fifth and sixth columns give the associated line strengths and upper energy levels. The final six columns in Table 1 list the observed LSR velocity and brightness temperature of each glycine line reported for each source. We have listed in boldface the LSR velocity and brightness temperature values for the lines that were displayed by Kuan et al. (2003).

\section{ESSENTIAL CRITERIA FOR ESTABLISHING THE IDENTIFICATION OF A NEW INTERSTELLAR MOLECULE}

The task of establishing the identification of a new interstellar molecule in a dense interstellar cloud is made more complicated by high spectral line densities. For example, at $3 \mathrm{~mm}$ wavelength Sgr B2(N-LMH) has a spectral line density of 6.06 lines per $100 \mathrm{MHz}$, half of which are unidentified (Friedel et al. 2004). This is the highest reported line density in radio astronomy. Hence, the detection of spectral lines with frequencies close to a new species is not in itself sufficient evidence for a correct assignment. Consequently, there are several criteria to consider in securing the correct identification of a new interstellar molecule such as glycine.

Rest Frequencies: The most important criterion for establishing the identification of a new interstellar molecule is that the rest frequencies must be established to a high degree of accuracy. Preferably, the spectral line astronomical rest frequencies to be used in the interstellar search should have been directly measured in the laboratory. If such direct measurements are not available, interpolation fitting of the available laboratory data from other frequencies should allow construction of a high precision Hamiltonian model which can be used to predict search frequencies with uncertainties on the order of 1 part in 10 million.

Frequency Agreement: There must be frequency agreement among all detected transitions. This means that an accurate astronomical rest frequency of the assigned transition must be in reasonable agreement with the frequency corresponding to the LSR velocity of the source. Variations in the LSR velocity of a given molecular species can occur when regions with different kinematics contribute to the emission or absorption spectrum. However, it is evident that emission lines from a given molecular species which emanate from a region 
with no large kinematic variance will have a well-defined velocity field. Examples are the VLA measurements of the kinematics of methyl formate $\left(\mathrm{HCOOCH}_{3}\right)$ toward OMC-1 by Hollis et al. (2003a) and ethyl cyanide $\left(\mathrm{CH}_{3} \mathrm{CH}_{2} \mathrm{CN}\right)$ toward $\mathrm{Sgr}$ B2(N-LMH) by Hollis et al. (2003b). If there is a source velocity gradient, as established from observed transitions of known molecules, it cannot be a random function of transition frequency or energy level.

Furthermore, it is not uncommon to have an interstellar spectral line from one species blend with another line of either the same or another species. In order to correctly assign or confirm a line identification, a common standard is that the lines should be at least resolved by the Rayleigh criterion. That is, the minimum distance between two spectral lines in frequency space must be such that the maximum intensity of one line falls on the first null of the other (see, for example, Jenkins \& White 1957; Sommerfeld 1964). This is approximately equivalent to requiring that overlapping lines be separated by their full line width at half maximum intensity (assuming identical line profiles). In practice, a more stringent criterion based on the signal-to-noise ratio is often needed whereby two overlapping lines can be considered resolved if they are at least separated at half-maximum intensity of the weakest line.

Beam Dilution: Suppose one uses a single-element radio telescope with circular aperture of diameter D (such as the NRAO $12 \mathrm{~m}$ ) to observe separate transitions of some molecule at $50 \mathrm{GHz}, 100 \mathrm{GHz}$, and $150 \mathrm{GHz}$. The full half-power beam width (HPBW) of the telescope is approximately given by $1.22 \lambda / \mathrm{D}$ rad (Born \& Wolf 1980; see also Rohlfs \& Wilson 2000), so in this case the telescope HPBW changes by a factor of 3 over the range of observations. As we will discuss, beam dilution scales with source size squared and beam size squared. To examine the required correction for beam dilution, we can start with $\left\langle N_{T}\right\rangle$, the beamaveraged molecular column density determined from the integrated intensity of a rotation transition observed by a single-element radio telescope. In autocorrelation mode, with the assumptions of LTE and low optical depth ${ }^{9}$ (see, for example, Snyder et al. 2001; Dickens et al. 1997), $<N_{T}>$ is given by

$$
<N_{T}>=\frac{1.67 W_{\mathrm{T}} Z e^{E_{\mathrm{u}} / T_{\mathrm{rot}}}}{S \mu^{2} \nu} \times 10^{14} \mathrm{~cm}^{-2}
$$

\footnotetext{
${ }^{9}$ The corrections to apply when the optical depth is not low and when the excitation is subthermal have been discussed by in detail by Goldsmith \& Langer (1999) and are mentioned in Appendix A. The aspects of maser excitation have been examined by Reid \& Moran (1988). Because we are following the assumptions of Kuan et al. (2003) that their reported glycine lines are optically thin and in LTE, we will confine our discussion to this particular case.
} 
In equation (1), $W_{\mathrm{T}}=\int T_{R} d v$ in $\mathrm{K} \mathrm{km} \mathrm{s}^{-1}$, where $\mathrm{T}_{R}$ is the radiation temperature of the source and $d v$ is the FWHM line width. $\mathrm{T}_{R}$ is related to the measured antenna temperature $T_{R}^{*}$ (corrected for atmospheric attenuation, rear spillover, blockage, ohmic losses, and forward spillover) by $\mathrm{T}_{R}=T_{R}^{*} / \eta_{C}$, where $\eta_{C}$ is the source-beam coupling efficiency (Kutner \& Ulich 1981). Other terms in equation (1) are $Z$, the rotational partition function, $\mathrm{E}_{\mathrm{u}}$, the upper rotational energy level, $\mathrm{T}_{\text {rot }}$, the rotational temperature, $\mathrm{S}$, the line strength, $\mu^{2}$, the square of the dipole moment in Debye ${ }^{2}$, and $\nu$, the frequency in GHz. If the source is comparable to or smaller than the main beam, $\eta_{C}=\eta_{M}^{*} \mathrm{~B}$, where $\eta_{M}^{*}$ is the corrected main beam efficiency and is the fraction of power in the main beam relative to the power in the main beam plus error beam (Jewell 1990). B is the geometric coupling efficiency between the main beam and the source, and is often called the beam filling factor. Note that $\eta_{M}^{*}$ is related to the phase errors of the reflector surface and is thus a function of frequency. For example, for the NRAO $12 \mathrm{~m}, \eta_{M}^{*}=0.83$ at $113 \mathrm{GHz}$ and 0.45 at $240 \mathrm{GHz}$. The above formalism is applicable to sources with angular extent smaller than or on the order of the main beam. Highly extended sources may couple with some or all of the error beam and require a different source-beam convolution.

For a circular Gaussian telescope beam size $\Theta_{b}$ centered on the peak of a circular Gaussian source of size $\Theta_{s}$, the resulting convolution yields a beam filling factor B given by (e.g., see equation (28) of Ulich \& Haas 1976)

$$
B=\frac{\Theta_{s}^{2}}{\Theta_{b}^{2}+\Theta_{s}^{2}} .
$$

If $\Theta_{s} \gg \Theta_{b}, \mathrm{~B} \sim 1$. When $\Theta_{s} \sim \Theta_{b}, \mathrm{~B} \sim 0.5$, but if $\Theta_{s} \ll \Theta_{b}, \mathrm{~B} \sim \frac{\Theta_{s}^{2}}{\Theta_{b}^{2}}$.

Relative Intensities: Once several molecular transition assignments have been made, their relative intensities must be tested for consistency. For a source comparable to or smaller in angular size than the main beam of the telescope, the relative intensity of two single lines is determined by the corrected beam efficiencies, beam filling factors, dipole moments, line strengths, frequencies, energy levels, and rotational temperature according to equation (1) as

$$
\frac{T_{R}^{*}(i)}{T_{R}^{*}(j)}=\left(\frac{\eta_{M}^{*}(i)}{\eta_{M}^{*}(j)}\right)\left(\frac{B_{i}}{B_{j}}\right)\left(\frac{\nu_{i}}{\nu_{j}}\right)\left(\frac{S_{i}}{S_{j}}\right)\left(\frac{\mu_{i}^{2}}{\mu_{j}^{2}}\right) e^{-\frac{E_{u i}-E_{u j}}{T_{r o t}}} .
$$

Conformer I glycine has an a-type dipole moment, $\mu_{a}=0.911(6) \mathrm{D}\left(3.039(20) \times 10^{-30} \mathrm{C} \mathrm{m}\right)$, and a b-type, $\mu_{b}=0.697(10) \mathrm{D}\left(2.325(34) \times 10^{-30} \mathrm{C} \mathrm{m}\right)$ (Lovas et al. 1995). Therefore, for a given frequency, the product $\mathrm{S} \mu^{2}$ dictates that a-type transitions are stronger than b-type 
when $\mathrm{S}$ values are comparable.

A typical property of prolate asymmetric rotors such as glycine is that two low $\mathrm{K}_{-1}$ a-type and two b-type transitions become degenerate as the transition frequencies increase. In this four-fold degeneracy, all four transitions have the same rest frequency and energy levels, but different line strengths for the a-type and the b-type. This effect begins at $\sim$ $107 \mathrm{GHz}$ for glycine and it is important because the lines with this degeneracy are the most likely to be detected. The total intensity of a four-fold degenerate line at frequency $\nu_{i}$ is given by the sum of the four individual unresolved components, $\left(\sum_{k=1}^{4} T_{R}^{*}(k)\right)_{i}$, and the ratio of the $i t h$ degenerate line to the $j$ th degenerate line is given by equation (1) as

$$
\frac{\left(\sum_{k=1}^{4} T_{R}^{*}(k)\right)_{i}}{\left(\sum_{k=1}^{4} T_{R}^{*}(k)\right)_{j}}=\left(\frac{\eta_{M}^{*}(i)}{\eta_{M}^{*}(j)}\right)\left(\frac{B_{i}}{B_{j}}\right)\left(\frac{\nu_{i}}{\nu_{j}}\right)\left(\frac{S_{a i} \mu_{a}^{2}+S_{b i} \mu_{b}^{2}}{S_{a j} \mu_{a}^{2}+S_{b j} \mu_{b}^{2}}\right) e^{-\frac{E_{u i}-E_{u j}}{T_{r o t}}}
$$

Similar expressions can be derived for comparing the intensity of a single glycine line to either a two-fold or a four-fold degenerate glycine line.

Confirming Transitions: Once a candidate line is assigned, the assumption of optical thinness under LTE conditions allows predictions of intensities of additional confirming transitions unless line self-absorption, maser activity, or some other mitigating effect is evident. Hence, a key test of the correctness of the assignment of a transition is that any other transitions connected by favorable transition probabilities must also be present if the relative intensity predictions lead to detectable signal levels.

The maximum line strength for a-type asymmetric rotor R-branch transitions of a molecule like glycine occurs when $\mathrm{S}$ approaches $\mathrm{J}^{\prime}$, the upper level value of J. For a-type transitions, this means that the strongest transitions in a series will be those for which

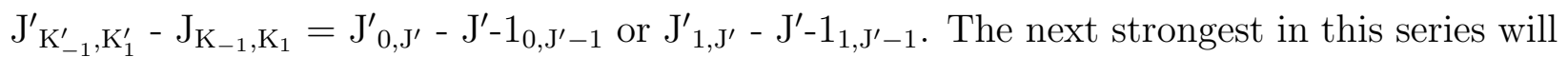
be $\mathrm{J}_{1, \mathrm{~J}^{\prime}-1}^{\prime}-\mathrm{J}^{\prime}-1_{1, \mathrm{~J}^{\prime}-2}$ or $\mathrm{J}_{2, \mathrm{~J}^{\prime}-1}^{\prime}-\mathrm{J}^{\prime}-1_{2, \mathrm{~J}^{\prime}-2}$. For b-type transitions, the maximum line strength occurs when $\mathrm{S}$ approaches $\mathrm{J}^{\prime}-1$. Here the strongest transitions will be those for which $\mathrm{J}_{0, \mathrm{~J}^{\prime}}$ - $\mathrm{J}^{\prime}-1_{1, \mathrm{~J}^{\prime}-1}$ or $\mathrm{J}_{1, \mathrm{~J}^{\prime}}^{\prime}-\mathrm{J}^{\prime}-1_{0, \mathrm{~J}^{\prime}-1}$. The next strongest will be $\mathrm{J}_{1, \mathrm{~J}^{\prime}-1}^{\prime}-\mathrm{J}^{\prime}-1_{2, \mathrm{~J}^{\prime}-2}$ or $\mathrm{J}_{2, \mathrm{~J}^{\prime}-1}^{\prime}-\mathrm{J}^{\prime}-$ $1_{1, J^{\prime}-2}$. Note that a-type transitions follow radiative selection rules where the parity change of the quantum numbers $\mathrm{K}_{-1}, \mathrm{~K}_{1}$ in a transition is e,e $\leftrightarrow$ e,o (even, even $\leftrightarrow$ even, odd) or o,o $\leftrightarrow$ o,e while the b-type transitions obey e,e $\leftrightarrow$ o,o or e,o $\leftrightarrow$ o,e (see, for example, Townes \& Schawlow 1975). 


\section{AN EXAMINATION OF THE DATA}

Kuan et al. (2003) state that their 27 glycine lines (Table 1) are detected in one or more hot molecular cores (HMCs). We note that HMCs are compact objects where interferometric measurements have shown that structurally similar but simpler large molecules are concentrated with diameters $<10$ ". For example, the HMC of one of their sources, Sgr B2(N-LMH), is $\sim 4 "$ as measured in ethyl cyanide $\left(\mathrm{CH}_{3} \mathrm{CH}_{2} \mathrm{CN}\right)$ and vinyl cyanide $\left(\mathrm{CH}_{2} \mathrm{CHCN}\right)$ by Liu \& Snyder (1999). On the other hand, Kuan et al. (2003) assume that their HMC glycine lines are optically thin and in LTE and do not correct for beam dilution in their rotational temperature diagrams- a tacit assumption that their glycine sources are extended enough to at least fill their beam. In the case of Orion KL, they speculated that the glycine emission is extended because the rotational temperature diagram has a "tight fit" and, in addition, perhaps the emission is not perfectly centered at the nominal compact ridge position. They used this scenario to reject the Orion glycine negative results found by Combes et al. (1996) with the IRAM $30 \mathrm{~m}$ telescope because the narrower $30 \mathrm{~m}$ beam might have missed a significant portion of the glycine emission observed by the wider NRAO $12 \mathrm{~m}$ beam.

We note that equation (1) of Kuan et al.(2003) is basically the same as equation (23) of Goldsmith \& Langer (1999) if the gas is assumed to be optically thin in LTE. The resulting column density is called a beam-averaged total column density. However, equation (38) of Goldsmith \& Langer (1999) shows that equation (1) actually is based on the further assumption that each glycine source fills the beam at each of the reported glycine frequencies. Otherwise, the derived glycine beam-averaged total column densities and rotational excitation temperatures have little physical meaning; they just become numerical parameters of a least-squares fit with little predictive value. How can the condition that the reported glycine sources fill each beam be satisfied? Kuan et al. (2003) state that their half-power beamwidths are $\sim 30 ", 45 "$, and $60 "$ at $1.3,2$, and $3 \mathrm{~mm}$, respectively. Table 1 of Kuan et al. (2003) lists the $2 \mathrm{~mm}$ lines (starting with glycine line 1) as the lowest frequency glycine lines that they claim. Thus these lines would determine the smallest extent $(\sim 45$ ") that the reported glycine could have in equation (1) of Kuan et al. (2003) without suffering from beam dilution. Consequently, in our analysis we used 45" as our upper limit for our trial source size.

In the following, we will apply the criteria from $\S 3$ to examine available observational data for the possibility that the spectral lines assigned to glycine have been misidentified. In order to address the ambiguities introduced by the glycine source sizes, we will assume two cases: (1) the glycine emission sources are HMCs with core diameters typically < 10"; or (2) glycine fills the beam of the $12 \mathrm{~m}$ telescope and hence is extended over at least 45 " in 
each source. For our analysis we will use our newly determined astronomical rest frequencies listed in Table 1.

\subsection{VLA Searches for Glycine in Compact Sources: Orion and Sgr B2(N-LMH)}

In 2001, Hollis et al. (2003b) conducted a deep Q-band ( $\lambda \sim 7 \mathrm{~mm}$ ) search with the Very Large Array (VLA) toward OMC-1 in four rotational transitions of conformer I glycine. The J2000.0 phase center for their observations was $\alpha=5^{h} 35^{m} 14^{s} .25$ and $\delta=-5^{\circ} 22^{\prime} 35^{\prime}$. 5 , which is midway between the Orion hot core source and the peak formic acid position of Liu et al. (2002). The Kuan et al. (2003) pointing position precessed from B1950.0 to J2000.0 was $\alpha$ $=5^{h} 35^{m} 14^{s} .48$ and $\delta=-5^{\circ} 22^{\prime} 36^{\prime \prime} .6$ toward Orion KL. The average $12 \mathrm{~m}$ telescope half-power beam width (HPBW) for the Orion observations of Kuan et al. (2003) was $\sim 41$ " and the VLA primary beam $\sim 45 "$, so the difference in pointing positions is not significant. The best VLA upper limits of Hollis et al. (2003b) were for the $7_{0,7}-6_{0,6}$ transition at $47,753.841(28)$ $\mathrm{MHz}$ with $\mathrm{S}=6.851, \mathrm{E}_{\mathrm{u}}=8.657 \mathrm{~K}$, and synthesized telescope beam size $\Theta_{b}=6^{\prime \prime}:\left\langle N_{T}>\right.$ $<8.0 \times 10^{14} \mathrm{~cm}^{-2}$ for $T_{\text {rot }}=43 \mathrm{~K}$; and $\left\langle N_{T}><25.2 \times 10^{14} \mathrm{~cm}^{-2}\right.$ for $T_{\text {rot }}=100 \mathrm{~K}$. Kuan et al. (2003) found $\left\langle N_{T}\right\rangle=4.37 \times 10^{14} \mathrm{~cm}^{-2}$ for $T_{\text {rot }}=141 \mathrm{~K}$, which is below the upper limits of Hollis et al. (2003b) only if there is no significant beam dilution in the $12 \mathrm{~m}$ results for Orion KL. However, if the glycine source size is 6" in diameter (suggested by Hollis et al. 2003b), then the $12 \mathrm{~m}$ glycine column density $\left\langle N_{T}>\right.$ becomes $2.08 \times 10^{16} \mathrm{~cm}^{-2}$, which is far above the VLA upper limits.

In 2003, Hollis et al. (2003a) repeated their VLA Q-band glycine search toward Sgr B2(N$\mathrm{LMH}$ ). Again the results were negative, and the best upper limits were for the $7_{0,7}-6_{0,6}$

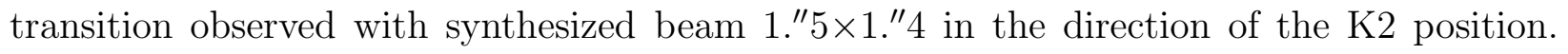
They reached an upper limit of $\left\langle N_{T}><1.4 \times 10^{17} \mathrm{~cm}^{-2}\right.$ for $T_{\text {rot }}=170 \mathrm{~K}$. In this same source, Kuan et al. (2003) reported $\left\langle N_{T}\right\rangle=4.16 \times 10^{14} \mathrm{~cm}^{-2}$ for $T_{\text {rot }}=76 \mathrm{~K}$, from observations with an average HPBW of $38^{\prime \prime}$. If the glycine source size is comparable to the VLA synthesized beam, then the $12 \mathrm{~m}$ glycine column density $<N_{T}>$ becomes $2.67 \times 10^{17}$ $\mathrm{cm}^{-2}$, which is again above the VLA upper limits.

In summary, the VLA glycine negative results (Hollis et al. 2003b; 2003a) contradict the glycine detection reports only if the Orion glycine sources in Orion and Sgr B2(N-LMH) are compact. However, as discussed previously, Kuan et al. (2003) assumed that their glycine emission sources are extended and at least 45 " in diameter. In the following sections, we will examine this assumption. 


\subsection{Search for Glycine in Extended Sources: Orion KL}

A direct way to examine the assumption of Kuan et al. (2003) that their reported glycine line sources are extended is to use their reported results to generate predicted intensities for other glycine lines that they did not observe. These predictions can be checked against other single-element telescope observations that would be sensitive to glycine emission from extended sources. In particular, we observed interstellar acetone $\left[\left(\mathrm{CH}_{3}\right)_{2} \mathrm{CO}\right]$ in 1995 March with the NRAO $12 \mathrm{~m}$ radio telescope toward Sgr B2(N) (Snyder et al. 2002). During Orion time, we searched for the nearly four-fold degenerate $\mathrm{J}=19-18$ glycine transitions around $113,336 \mathrm{MHz}$. Our Orion KL pointing position was essentially identical to that used by Kuan et al. (2003): exactly the same right ascension and within 9" in declination. The NRAO $3 \mathrm{~mm}$ single-sideband SIS receiver had sideband rejection $\geq 25 \mathrm{db}$ and effective system temperature (referenced to above the atmosphere and including rear and forward spillover efficiencies) of $\sim 300 \mathrm{~K}$. Chopper calibration corrected for atmospheric extinction and telescope losses and the resultant data are on the $\mathrm{T}_{R}^{*}$ temperature scale (Kutner and Ulich 1981). Data were taken while position switching $30^{\prime}$ in azimuth, while Kuan et al. (2003) switched by $20^{\prime}$. The half power beam width (FWHM) was $\sim 56^{\prime \prime}$ at $113.3 \mathrm{GHz}$. The spectrometer consisted of the NRAO Hybrid Spectrometer with two 256 channel filter banks used as a backup. The Hybrid Spectrometer was operated with two polarization IFs in parallel, with $300 \mathrm{MHz}$ bandwidth and 768 Hanning-smoothed channels per IF, giving an effective spectral resolution of $0.781 \mathrm{MHz}$. The filter banks were operated with two IFs in parallel, with $250 \mathrm{kHz}$ resolution in one bank and $500 \mathrm{kHz}$ in the other. Therefore, this system was exactly the same as that used by Kuan et al. (2003) except that they chose to use wider filters for their observations. The NRAO $12 \mathrm{~m}$ data were reduced using the NRAO data reduction package UniPOPS (Salter, Maddalena, \& Garwood 1995) ${ }^{10}$.

The Kuan et al. (2003) pointing position toward Orion KL was $\alpha(\mathrm{B} 1950.0)=5^{h} 32^{m} 47^{s} .0$ and $\delta(\mathrm{B} 1950.0)=-5^{\circ} 24^{\prime} 30^{\prime \prime} .0$. Our pointing position was $\alpha(\mathrm{B} 1950.0)=5^{h} 32^{m} 47^{s} .0$ and $\delta(\mathrm{B} 1950.0)=-5^{\circ} 24^{\prime} 21^{\prime \prime} .0$, which in our $\sim 56$ " beam was only a negligible 9" difference. Table 2 summarizes our Orion KL search frequencies for the $\mathrm{J}=19-18$ glycine transitions and for the test lines used to check the operation of the system. The first column lists rest frequencies; the second and third list molecular identifications (when known) and rotational quantum numbers; the fourth, fifth, and sixth columns give the transition types, line strengths, and upper energy levels; and the next two columns give intensities and line widths for Orion KL. Figure 1 shows the Orion KL spectra from 113,323 to 113,354 MHz (centered at 113,339 $\mathrm{MHz}$ ) observed with the Hybrid Spectrometer on the NRAO $12 \mathrm{~m}$. The spectral positions

\footnotetext{
${ }^{10}$ UniPOPS information is available at http://www.gb.nrao.edu/ rmaddale/140ft/unipops/unipops_toc.html.
} 
of the negative results for the nearly four-fold degenerate $\mathrm{J}=19-18$ glycine lines (listed in Table 2) are marked by the four vertical lines. The unidentified line U113226 is on the left, and the $6_{1}-6_{0} \mathrm{E}_{1}$ transition of $\mathrm{CH}_{3} \mathrm{OD}$ is on the right; these two lines served as system checks.

Kuan et al.(2003) reported the detection of 15 optically thin glycine lines in the direction of Orion KL. If they were glycine, lines $1,5,6,8,9,14,15,16,17,18$, and 25 in Table 1 would be single transitions. Lines 11, 21, 24, and 26 would be four-fold degenerate. Of these 15 lines, none contained connecting confirming transitions, which would be a key test of the correctness of the glycine assignment as discussed in $\S 3$. Among these, we note that lines 5, 14, 15, 16, and 26 marginally meet the Rayleigh criterion for spectral resolution. By applying the discussion in $\S 3$ and adapting equation 4 to each of the reported Orion glycine lines, we can use each reported line to make an independent prediction of the intensity of the nearly four-fold degenerate $\mathrm{J}=19-18$ glycine lines that we did not detect, as shown in Figure 1. Figure 2 shows the range of predicted intensities that we should have observed with the NRAO $12 \mathrm{~m}$ telescope for each of the 15 of the reported Orion glycine transitions emanating from a 45" extended source. For each prediction, three different points are labeled to denote the Kuan et al. (2003) rotational temperature and its uncertainties, $\mathrm{T}_{\text {rot }}=141_{-37}^{+76}$ $\mathrm{K}$. The uncertainties for each rotational temperature point are based on the rms noise level given for each reported glycine line by Kuan et al. (2003) (see Table 1). The dotted line in Figure 2 shows the $3.7 \mathrm{mK}$ noise level from Figure 1. For example, Orion line 21 has a peak intensity $\mathrm{T}_{R}{ }^{*}=230 \pm 10.0 \mathrm{mK}$ as observed with a 30 " beam. In Table 1 , it is assigned as a degenerate quartet of two a-type and two b-type glycine lines at 206,468 MHz: $35_{0,35}-34_{0,34}$; $35_{1,35}-34_{1,34} ; 35_{0,35}-34_{1,34}$; and $35_{1,35}-34_{0,34}$. If line 21 is an optically thin glycine line in LTE with a rotational temperature of $141 \mathrm{~K}$, then the nearly blended $\mathrm{J}=19$ - 18 quartet would appear in Figure 1 with peak intensity $\mathrm{T}_{R}{ }^{*} \sim 143 \mathrm{mK} \pm 7 \mathrm{mK}$ for an extended source size of $\Theta_{s}=45$ ". All of the predictions plotted in Figure 2 are above the $3.7 \mathrm{mK}$ noise level from Figure 1. Hence, if the Orion reported glycine detection were correct, we should have detected a blended line with intensity $5 \mathrm{mK} \leq \mathrm{T}_{R}^{*} \leq 205 \mathrm{mK}$ for the $\mathrm{J}=19$ - 18 glycine quartet. Since we did not, we conclude that glycine has not been detected in an extended source in Orion KL. In $\S 4.1$, we ruled out the detection of glycine in a compact source in

Orion. Consequently, we conclude that the presence of interstellar glycine has not been verified in Orion KL.

\subsection{Search for Glycine in Extended Sources: W51}

During the 1995 March observations with the NRAO $12 \mathrm{~m}$, we also searched for the four-fold nearly degenerate $\mathrm{J}=19$ - 18 glycine transitions in the direction of W51. The 
Kuan et al. (2003) pointing position toward W51 e1/e2 was $\alpha(B 1950.0)=19^{h} 21^{m} 26^{s} .3$ and $\delta(\mathrm{B} 1950.0)=+14^{\circ} 24^{\prime} 39^{\prime \prime} .0$. Our W51 pointing position was $\alpha(\mathrm{B} 1950.0)=19^{h} 21^{m} 26^{s} .3$ and $\delta(\mathrm{B} 1950.0)=+14^{\circ} 24^{\prime} 43^{\prime \prime} .0$, which in our $\sim 56^{\prime \prime}$ beam was only a negligible $4 "$ difference. As with the Orion KL observations, our W51 data were taken while position switching $30^{\prime}$ in azimuth, while Kuan et al. (2003) switched $20^{\prime}$. Again, the system was exactly the same as that used by Kuan et al. (2003) except that they used wider filters. In Table 2, the last two columns give intensities and line widths for the W51 search frequencies for the $\mathrm{J}=19$-18 glycine transitions and for the test lines used to check the operation of the system; they are the same transitions used for the Orion KL search. Figure 3 shows the W51 spectra from 113,323 to $113,354 \mathrm{MHz}$ (centered at 113,339 MHz) observed with the Hybrid Spectrometer on the NRAO $12 \mathrm{~m}$. As in the case of Orion KL, the spectral positions of the negative results for the nearly four-fold degenerate $\mathrm{J}=19-18$ glycine lines (listed in Table 2) are marked by the four vertical lines centered at 113,336 MHz. As in Figure 1, the system checks are the unidentified line U113226 on the left, and the weak $6_{1}-6_{0} \mathrm{E}_{1}$ transition of $\mathrm{CH}_{3} \mathrm{OD}$ on the right.

Kuan et al. (2003) reported the detection of 16 optically thin glycine lines in the direction of W51. If these lines were glycine, lines 3, 4, 5, 7, 8, 9, 13, 15, and 25 in Table 1 would be single transitions. Line 12 would be two-fold degenerate and lines 19, 20, 21, 24,26 , and 27 would be four-fold degenerate. Of these 16 lines, none contained connecting confirming transitions, which would be a key test of the correctness of the glycine assignment as discussed in $\S 3$. Among the displayed spectra, line 27 does not meet the Rayleigh criterion for spectral resolution. As with the Orion KL data, we can use the intensity of each of the reported glycine lines to independently predict the intensity of the nearly four-fold degenerate $\mathrm{J}=19-18$ glycine lines at $113,366 \mathrm{MHz}$ that we did not detect, as shown in Figure 3. Figure 4 shows the range of predicted intensities that we should have observed with the NRAO $12 \mathrm{~m}$ telescope for each of the 16 reported W51 glycine transitions emanating from a 45" extended source. As in Figure 2, three different points are labeled for each prediction to denote the Kuan et al. (2003) rotational temperature and its uncertainties, $\mathrm{T}_{\text {rot }}=121_{-32}^{+71}$ $\mathrm{K}$. The uncertainties for each rotational temperature point are based on the rms noise level given for each reported glycine line by Kuan et al. (2003) (see Table 1). The dotted line in Figure 4 shows the $6.4 \mathrm{mK}$ noise level from Figure 3. All of the predictions plotted in Figure 4, except those from line 19, are above the this noise level. Hence, the intensities of 15 out of 16 glycine lines predict that we should have detected a blended $\mathrm{J}=19$ - 18 glycine line quartet with intensity $10 \mathrm{mK} \leq \mathrm{T}_{R}^{*} \leq 105 \mathrm{mK}$ if the reported W51 glycine detection is correct. Since we did not, we conclude that the presence of interstellar glycine has not been verified in W51. 


\subsection{Search for Glycine in Extended Sources: Sgr B2(N-LMH)}

Kuan et al. (2003) assigned glycine transitions to 13 emission lines in the direction of Sgr B2(N-LMH) at $\alpha(\mathrm{B} 1950.0)=17^{h} 44^{m} 10^{s} .20$ and $\delta(\mathrm{B} 1950.0)=-28^{\circ} 21^{\prime} 15^{\prime \prime} .0$. Their rest frequencies were calculated with respect to $\mathrm{V}_{\mathrm{LSR}}=64 \mathrm{~km} \mathrm{~s}^{-1}$. Their emission lines 1, 2, 6, and 7 in Table 1 were assigned to single glycine transitions. Line 10 would have a five-fold degeneracy and line 12 a two-fold if they were glycine. Lines 19, 20, 21, 22, 23, 24 , and 26 would be four-fold degenerate. Of these 13 lines, only lines 21 and 22 would contain connecting confirming transitions, a key test of the correctness of the assignment as discussed in $\S 3$. Only lines 19, 20, 21, 23, and 26 were displayed by Kuan et al. (2003); of these, lines 19, 21, and 23 marginally meet the Rayleigh criterion for spectral resolution. Fortunately, Nummelin et al. (1998) surveyed Sgr B2(N) in the direction $\alpha(B 1950.0)=$ $17^{h} 44^{m} 10^{s} .10$ and $\delta(\mathrm{B} 1950.0)=-28^{\circ} 21^{\prime} 17^{\prime \prime} .0$ between 218.3 and $263.55 \mathrm{GHz}$ with the $15 \mathrm{~m}$ Swedish ESO-Submillimetre Telescope (SEST). Their position coincided with the Sgr B2(NLMH) position used by Kuan et al. (2003). Their beam width was $\sim 20$ " (adequate for extended sources), and their spectral resolution was $\sim 1.4 \mathrm{MHz}$, which corresponds to $\sim 1.8$ $\mathrm{km} \mathrm{s}^{-1}$ at $230 \mathrm{GHz}$. Their frequency range covered several important glycine transitions, which we will discuss. Rest frequencies were calculated with respect to $\mathrm{V}_{\mathrm{LSR}}=62 \mathrm{~km} \mathrm{~s}^{-1}$.

By applying the discussion in $\S 3$ to predict the expected glycine intensities, it is straightforward to use the Nummelin et al. (1998) data to examine the Sgr B2(N-LMH) glycine assignments of Kuan et al. (2003). If lines 26 and 27 were glycine, for any reasonable temperature their line strengths would make them the the two strongest glycine lines listed in Table 1. Line 26 was observed by Kuan et al. (2003) with the NRAO $12 \mathrm{~m}$ telescope to have $\mathrm{T}_{R}^{*}=94 \mathrm{mK}$. It was assigned to a degenerate glycine quartet consisting of two a-type and two b-type transitions with $\mathrm{J}=40-39, \mathrm{~K}_{-1}=1$ or 2 at $240,899.5 \mathrm{MHz}$. Nummelin et al. (1998) detected this line with SEST; it has a main beam brightness temperature $\mathrm{T}_{m b}$ $=300 \mathrm{mK}$. Kuan et al. (2003) assigned line 27 to a degenerate glycine quartet with $\mathrm{J}=$ $41-40, \mathrm{~K}_{-1}=0$ or 1 at $241,373.3 \mathrm{MHz}$. While line 27 would be expected to be slightly stronger than line 26, it was reported by Kuan et al. (2003) to be masked by unidentified interlopers in their Sgr B2(N-LMH) 12m data and the spectrum was not displayed. However, this interloper masking argument is not supported by the data of Nummelin et al. (1998), which show not only that the line 27 frequency falls in a relatively clear spectral region in Sgr B2(N-LMH), but also that line 27 was not detected (as we will discuss).

Nummelin et al. (1998) reported all of their data in units of $\mathrm{K}$ on the main beam brightness temperature $\left(\mathrm{T}_{m b}\right)$ scale. Hence, $\eta_{M}^{*}$, the main beam efficiency has already been taken into account, and equation 4 may be used for intensity predictions with $\mathrm{T}_{m b}$ substituted for $T_{R}^{*} / \eta_{M}^{*}$. To conduct our analysis, we used the $\mathrm{T}_{m b}=300 \mathrm{mK}$ intensity (with an 
estimated $50 \mathrm{mK}$ rms noise level uncertainty) of line 26 from Nummelin et al. (2003) as a basis for predicting the intensities of those $\mathrm{K}_{-1}=1$ or 2 degenerate glycine quartet lines connected to line 26 via two a-type and two b-type transitions. By confining our predictions to the Nummelin et al. (1998) data set, we eliminated any problems caused by calibration differences between the NRAO $12 \mathrm{~m}$ and SEST telescopes. Table 3 lists all of the $\mathrm{K}_{-1}=1$ or 2 degenerate glycine lines that fall in the $218.3-263.55 \mathrm{GHz}$ range of the Nummelin et al. (1998) survey that would be radiatively connected to line 26 if its assignment to the degenerate glycine quartet of two a-type and two b-type transitions with $\mathrm{J}=40-39, \mathrm{~K}_{-1}=1$ or 2 , were correct. The first column lists the individual quantum numbers of each degenerate frequency group, the second lists the transition type, and the third lists the rest frequency of each degenerate glycine line. Note that the line quartets are arranged in descending order of frequency. The top transition in each degenerate quartet feeds directly into the top transition in the next lower frequency degenerate quartet, the second transition in each degenerate quartet feeds directly into the second transition in the next lower degenerate quartet, etc. The fourth column in Table 3 gives the line strengths and the fifth the upper state energy levels. The next column gives the predicted values of $\mathrm{T}_{m b}$ for each line if the assignment of line 26 to the $\mathrm{J}=40-39, \mathrm{~K}_{-1}=1$ or 2 , degenerate glycine transition at $240,899.5 \mathrm{MHz}$ were correct. These predicted $\mathrm{T}_{m b}$ values (as well as the values for the upper and lower error bars) were calculated for a gas rotational temperature of $75 \mathrm{~K}$ with an uncertainty ranging from $59 \mathrm{~K}$ to $104 \mathrm{~K}$ (Kuan et al. 2003) in addition to the $50 \mathrm{mK}$ rms noise level uncertainty for line 26. The next column lists what was observed by Nummelin et al. (1998) at each frequency: the peak $\mathrm{T}_{m b}$ value for any clearly resolved line; the upper limit to the noise level if no line is present; or the notation ... indicating that the line is masked by the wings of an interloper line. The final column in Table 3 lists relevant comments, if any, for each observed frequency. Table 4 follows the format of Table 3 for the stronger $\mathrm{K}_{-1}=0$ or 1 degenerate glycine lines that fall in the same range of the Nummelin et al. (1998) survey. Again, the predicted intensities in Table 4 are based on the observed intensity of $\mathrm{T}_{m b}=300 \mathrm{mK}$ for line 26 (with an estimated $50 \mathrm{mK}$ rms noise level uncertainty) and the assumption that it can be assigned to glycine.

Examination of Table 3 shows that the $\mathrm{K}_{-1}=1$ or 2 frequencies of the degenerate glycine quartets $\mathrm{J}=43-42,38-37$, and $37-36$ are obscured by the wings of lines previously identified by Nummelin et al. (1998). U235.085 at 235,085 MHz would be a possible candidate for the $\mathrm{J}=39-38, \mathrm{~K}_{-1}=1$ or 2 , degenerate glycine transition at 235,084.8 MHz, but the peak intensity is low. Finally, the glycine lines missing from the Nummelin et al. (1998) data at the $\sim 100 \mathrm{mK}$ level are the $\mathrm{K}_{-1}=1$ or 2 frequencies of the degenerate glycine quartets $\mathrm{J}=42-41$ at 252,531.295 $\mathrm{MHz}$ and $41-40$ at 246,716.164 $\mathrm{MHz}$. The data for these missing lines and for line 26 at 240,900.647 MHz are shown in 
Figures 5(a), 5(b), and 5(c), adapted from the Sgr B2(N) data and molecular assignments of Nummelin et al. (1998).

Table 4 shows that the $\mathrm{K}_{-1}=0$ or 1 frequencies of the degenerate glycine quartets $\mathrm{J}=40-39,39-38$, and $38-37$ are obscured by the wings of lines previously identified by Nummelin et al. (1998). In addition, the spectral region around the degenerate glycine transition $\mathrm{J}=44-43$ at $258,820.597 \mathrm{MHz}$ is dominated by what appears to be narrow, weak interference spikes in the Nummelin et al. (1998) data for Sgr B2(N), Sgr B2(M), and Sgr B2(NW). The $\mathrm{K}_{-1}=0$ or 1 glycine lines missing from the Nummelin et al. (1998) data at the $\sim 50 \mathrm{mK}$ level are the $\mathrm{J}=43-42$ degenerate quartet at 253,005.255 MHz. Furthermore, the $\mathrm{J}=42-41$ degenerate quartet at $247,189.500 \mathrm{MHz}$ and the $\mathrm{J}=41-40$ degenerate quartet at 241,373.340 MHz (line 27 of Kuan et al. 2003) are missing at the $100 \mathrm{mK}$ level. The data for these missing lines are shown in Figures 5(d), 5(e), and 5(f).

Because of the five missing key quartets summarized in Tables 3 and 4 and shown in Figure 5, we conclude that glycine has not been detected in an extended source in Sgr B2(N). In $\S 4.1$, we ruled out the detection of glycine in a compact source in Sgr B2(N-LMH). Thus, we conclude that the presence of interstellar glycine has not been verified in Sgr B2(N).

\subsection{Alternative Identifications}

Now that we have shown that the carriers of key spectral lines assigned to glycine by Kuan et al. (2003) can not be verified as interstellar glycine, in this section we suggest some logical assignments to other, more common species. Our proposed carriers, listed in Table 5, are presented to underline the impact of line confusion on the growing and difficult problem of identifying new interstellar species. The first and second columns in Table 5 list the line number and rest frequency for glycine taken from Kuan et al. (2003). We list these older, less accurate glycine frequencies because they were the only rest frequencies reported by Kuan et al. (2003) for their detected lines. They reported that for most of their observations, filter bank spectrometers with 1 and $2 \mathrm{MHz}$ resolution were used in parallel mode, but filter banks of $1 \mathrm{MHz}$ and $500 \mathrm{kHz}$ resolution were used for all observations taken from 2000 December to 2001 March. However, it is not always clear which spectral resolution applies to each reported glycine line since most of their data were not displayed. Therefore, unless otherwise stated, we will assume a minimum uncertainty of $1 \mathrm{MHz}$ on top of the $2 \sigma$ computational uncertainty in the reported rest frequency of each line. For convenient comparison, the third column in Table 5 lists our newer, more accurate glycine frequencies taken from Table 1 of this paper. The fourth, fifth, and sixth columns list the possible molecular candidates, their quantum numbers, and the rest frequencies of their transitions, respectively. In $\S 1$, we pointed out that 
one of the peculiarities in the glycine detection report of Kuan et al. (2003) was that each of their three glycine sources (Orion KL, W51 e1/e2, and Sgr B2(N-LMH)) was observed to contain no more than 13 to 16 of the 27 independent spectral lines features and only 3 of the 27 spectral line features were found to be common to all three sources: lines 21, 24, and 26 in Table 1. Consequently, we will begin with a discussion of these lines.

Line 21 was observed with the NRAO 12 m telescope with a 30" beam, and was misassigned to the four-fold degenerate glycine quartet $35_{0,35}-34_{1,34}, 35_{1,35}-34_{1,34}, 35_{0,35}-34_{0,34}$, and $35_{1,35}-34_{0,34}$ at $206,468 \mathrm{MHz}$. In all 3 sources, line 21 is anomalously strong relative to all other purported glycine transitions that were observed. This suggests that even in the unlikely event that all the other lines reported by Kuan et al. (2003) belong to the same species, then line 21 most likely belongs to another molecular species altogether. Recently, vibrationally excited ethyl cyanide $\left(\mathrm{CH}_{3} \mathrm{CH}_{2} \mathrm{CN}\right)$ has been shown to be a common constituent of Sgr B2(N-LMH) (Mehringer et al. 2004). Furthermore, the $23_{6,18}-22_{6,17}$ A and $\mathrm{E}$ and $23_{6,17}-22_{6,16} \mathrm{~A}$ and $\mathrm{E}$ transitions of vibrationally excited ethyl cyanide $\left(\nu_{\mathrm{b}}=1\right)$ span the range of 206,466.6 to 206,467.3 MHz (J. C. Pearson, private communication 2003). Of particular interest are the $23_{6,18}-22_{6,17} \mathrm{~A}$ and $23_{6,17}-22_{6,16} \mathrm{~A}$ transitions listed in Table 1 because they are at most only $0.85 \mathrm{MHz}\left(1.3 \mathrm{~km} \mathrm{~s}^{-1}\right)$ away from the assumed glycine search frequency of Kuan et al. (2003). Therefore, we suggest that a possible carrier of line 21 is vibrationally excited ethyl cyanide. If this suggestion is correct, the slightly weaker ethyl cyanide $23_{6,18}-22_{6,17} \mathrm{E}$ and $23_{6,17}-22_{6,16} \mathrm{E}\left(\nu_{\mathrm{b}}=1\right)$ transitions at 206,466.623(8) MHz and 206,466.939(7) MHz, respectively, are blended into the low frequency shoulder of line 21 in Sgr B2(N-LMH), Orion KL, and W51 e1/e2 (see figures 1(a), (b), and (c) of Kuan et al. 2003).

Line 24 was misassigned to the nearly four-fold degenerate glycine quartet $36_{3,33}-35_{4,32}$, $36_{4,33}-35_{4,32}, 36_{3,33}-35_{3,32}$, and $36_{4,33}-35_{3,32}$ at an average observed frequency of $228,419.1$ MHz. Kuan et al. (2003) dismissed the frequency coincidence of the $\mathrm{J}=24-23, \mathrm{~K}_{-1}=11$ transition doublet of vinyl cyanide $\left(\mathrm{CH}_{2} \mathrm{CHCN}\right)$ as an "unlikely" $\nu=2$ state of the molecule. However, these transitions are actually in the out-of-plane bending mode, $\nu_{15}=1$, which Nummelin \& Bergman (1999) have shown to be the carrier of 45 lines of vinyl cyanide in Sgr B2(N) (including the vinyl cyanide doublet which was misassigned as line 24 of glycine). In their Table 40, Nummelin et al. (1998) list the rest frequency of the vinyl cyanide doublet as 228,417.8 MHz, but a new calculation by Lovas (2004) gives 228,418.10(17) MHz. As shown in Table 5 , the new vinyl cyanide rest frequency is only $\sim 1 \mathrm{MHz}$ away from the search frequency which was used by Kuan et al. (2003) for glycine, but it is $\sim 2 \mathrm{MHz}$ away from the new average glycine rest frequency.

The third key line, line 26 was misassigned to the four-fold degenerate glycine quartet 
$40_{1,39}-39_{2,38}, 40_{2,39}-39_{2,38}, 40_{1,39}-39_{1,38}$, and $40_{2,39}-39_{1,38}$ at an average observed frequency of $240,899.6 \mathrm{MHz}$, whereas the actual glycine frequency is $1.1 \mathrm{Mhz}$ higher (see Table 5). The Sgr B2(N) data for line 26 have been published by both Kuan et al. (2003) in their Figure 1d and by Nummelin et al. (1998) in their Figure 1. We note that line 26 has the same flux ( 6 Jy) in the $\sim 20$ " beam of Nummelin et al. (1998) as it has in the $\sim 26$ " beam of Kuan et al. (2003). This suggests that the carrier of line 26 could also be a constituent of a somewhat compact source, such as a hot molecular core.

In addition to lines 21,24, and 26, there are potential carriers for other reported glycine lines that were detected in only one or two of the three reported glycine sources. Several potentially viable alternative identifications were listed by Kuan et al.(2003) as possible line carrier candidates, but dismissed without further consideration. These worthy candidates will be discussed in order by source and listed in the remaining sections of Table 5 .

Line 12, reported in Sgr B2(N-LMH) and W51 e1/e2, was misassigned to the degenerate glycine pair $22_{12,11}-21_{12,10}$ and $22_{12,10}-21_{12,9}$ at an observed frequency of $150,909.8 \mathrm{MHz}$. $\mathrm{CH}_{2} \mathrm{DCH}_{2} \mathrm{CN}$ (Table 5) was considered and dismissed by Kuan et al. (2003) because of the combination of rare isotopic species (D) and an unlikely transition. However, because of the close frequency coincidence, it can not be completely eliminated from consideration without further observation of comparable transitions of that species.

Line 10, observed only in Sgr B2(N-LMH), was misassigned to the cluster of glycine lines $24_{1,23}-23_{2,22}, 24_{2,23}-23_{2,22}, 24_{1,23}-23_{1,22}, 22_{3,19}-21_{3,18}$, and $24_{2,23}-23_{1,22}$ at an average center frequency of $147,813.7 \mathrm{MHz}$ (Table 5). Kuan et al. (2003) recognized the possibility of overlap with the $21_{4,17}-20_{5,16}$ transition of $\mathrm{CH}_{3} \mathrm{CH}_{2} \mathrm{OH}$ (ethanol), but they misidentified it as a $\nu=1$ vibrationally excited state of ethanol and hence removed it from consideration as a possible identification. The correct assignment is to $\mathrm{g}-\mathrm{CH}_{3} \mathrm{CH}_{2} \mathrm{OH}$ (gauche ethanol) in the ground state (Table 5), so ethanol should remain in consideration as a possible carrier of line 10 .

Line 16, reported only in Orion KL, was misassigned to the $26_{2,24}-25_{2,23}$ transition of glycine at $164,870.0 \mathrm{MHz}$ (Table 5). This line is shown to be barely above the noise level by Kuan et al (2003) in their Figure 3. They recognized that the frequency of line 16 agrees almost perfectly with the degenerate transitions of $\left(\mathrm{CH}_{3}\right)_{2} \mathrm{CO}$ (acetone) listed in Table 5, but dismissed this possibility because the transitions are unfavorable. However, because of the close frequency agreement and the low intensity of line 16, there is no strong basis for ignoring acetone without further observations.

Line 13, reported only in W51 e1/e2, was misassigned to the $23_{6,18}-22_{6,17}$ transition of glycine at 160,153.458(266) MHz (Table 5). Line 13 was not reported in either Sgr B2(N- 
$\mathrm{LMH}$ ) or Orion, but the detection of U160144 was listed instead. No spectra of line 13 or U160144 were displayed by Kuan et al. (2003). Thus it is fortunate that Lee \& Cho (2002) used the TRAO $14 \mathrm{~m}$ telescope to conduct an Orion survey over this frequency range. They detected and identified the $8_{1,8}-7_{1,7}$ transition of $\mathrm{H}_{2} \mathrm{C}_{2} \mathrm{O}$ (ketene) at $160,142 \mathrm{MHz}$, with a high frequency shoulder. An examination of their published spectra leaves no doubt that U160144 in Orion is ketene, and the high frequency shoulder (which extends to almost 160,160 MHz) includes the frequency of line 13. A further complication is that line 13 is almost coincident with transitions of both vinyl cyanide $\left(\mathrm{CH}_{2} \mathrm{CHCN}\right)$ and acetone $\left(\left(\mathrm{CH}_{3}\right)_{2} \mathrm{CO}\right)$ (see Table 5). The vinyl cyanide line was considered and ruled out by Kuan et al. (2003) because it appeared to be an unfavorable transition in the $\nu=2$ vibrational state. However, it is actually in the $\nu_{15}=1$ state. Thus we have listed several possibilities for line 13: it could be a velocity component of ketene; it could be acetone; it could be vinyl cyanide; or it could be a partial mixture of these and unknown components. An observational check on these possibilities would be straightforward.

Probably there are other good alternative identifications for the additional lines reported by Kuan et al. (2003), due to the plethora of transitions from ground and excited states of many common interstellar molecules. For example, deep searches for acetone have only been conducted in Sgr B2(N) (Snyder et al. 2002), but acetone may well be detectable in Orion KL and W51 e1/e2 at the levels cited by Kuan et al. (2003). Certainly most of the good alternative candidates for lines reported as glycine are more likely to be detectable than glycine, and the identification of these candidates can be checked by further observational work on other favorable transitions.

\section{ROTATIONAL TEMPERATURE DIAGRAM LIMITATIONS}

In a given spectral range for a given source, it is our intention to demonstrate that one can use random $\mathrm{U}$ line data that are nearly frequency coincident with glycine transitions to obtain a reasonable looking rotational diagram. The results of the rotation diagram are then used to show that the most likely glycine transitions (particularly a-type with large transition strengths) are either severely lacking in predicted intensity or altogether missing in the given spectral range.

Kuan et al. (2003) used rotation temperature diagrams constructed from Sgr B2(N$\mathrm{LMH}$ ), Orion KL, and W51 e1/e2 data to bolster their arguments for the identification of glycine. In Appendix A, we have noted that rotational temperature diagrams cannot be used as independent tools to verify interstellar spectral line identifications because the frequency parameter in equation A4 or A5 will dominate the integrated intensity, line strength, and 
dipole moment terms for typical spectral line data in the millimeter or submillimeter range. In the following discussion, we illustrate this important point. As a data base, we used the 123 unidentified (U) lines detected in a millimeter wavelength spectral line survey conducted by Friedel et al. (2004) toward Sgr B2(N-LMH) with the BIMA Array. We searched this U line data base for near frequency-coincident transitions of glycine with the following criteria for matching lines:

1. The $2 \sigma$ frequency uncertainty of each calculated glycine transition had to be less than $2.82 \mathrm{MHz}$ (equivalent to twice the largest $1 \sigma$ uncertainty from Kuan et al. 2003). This uncertainty turned out to be less than $1.84 \mathrm{MHz}$.

2. Friedel et al. (2004) established the rest frequency of each $\mathrm{U}$ line by assuming a $\mathrm{v}_{L S R}$ of $64 \mathrm{~km} \mathrm{~s}^{-1}$. Kuan et al. (2003) states that an acceptable velocity range for Sgr B2(N$\mathrm{LMH}$ ) is between 58 and $75 \mathrm{~km} \mathrm{~s}^{-1}$. Therefore, we allowed a tolerance in our U line frequencies which corresponded to a $\mathrm{v}_{L S R}$ of $64_{-6}^{+11} \mathrm{~km} \mathrm{~s}^{-1}$ with an additional uncertainty of $\pm 390 \mathrm{kHz}$ due to the spectral resolution used by Friedel et al. (2004) for the $\mathrm{U}$ line observations.

3. The width of each matched $\mathrm{U}$ line had to be between 2.8 and $12.7 \mathrm{~km} \mathrm{~s}^{-1}$, which is the spread in line widths from the data in Kuan et al. (2003) toward Sgr B2(N). There were 2 exceptions, but the uncertainties easily reached into this range.

4. We selected only $U$ lines whose intensity was not too great for the associated transition. If the $\mathrm{U}$ line appeared to be a blend of transitions from several species, it was not used.

Table 6 lists the glycine and $U$ line frequencies that will be used in our rotational temperature diagram discussion. The first two columns list calculated glycine rest frequencies and corresponding transitions. The third and fourth columns list the product of the line strength and dipole moment squared $\left(S \mu^{2}\right)$ and upper energy $\left(E_{\mathrm{u}}\right)$ for each glycine transition. The fifth column lists $U$ lines which were selected using criteria listed above. The sixth and seventh columns list the U line integrated intensity derived from Friedel et al. (2004) and synthesized beam size employed in the observations. Table 6 shows that the frequencies of $11 \mathrm{U}$ lines (col. 5) nearly match frequencies of 12 degenerate and non-degenerate glycine transitions (col. 1). Under the assumption of no beam dilution, the glycine rotational temperature diagram in Figure 6 was constructed using calculated glycine frequencies in equation A4. The Figure 6 data points were fit with a straight line by a least squares method that weights each integrated intensity by its uncertainty which generally results in smaller uncertainties in slope and intercept when compared to an unweighted least squares fit (Bevington \& Robinson 
1992). The resulting weighted fit $^{11}$ produced a rotational temperature of $90(13) \mathrm{K}$ and a total column density, $\left\langle N_{T}>\right.$, of $1.1(1) \times 10^{17} \mathrm{~cm}^{-2}$. The derived rotational temperature and column density is similar to those of other molecules detected toward SgrB2(N-LMH) at high spatial resolution. If Figure 6 is a valid glycine rotational diagram, then the derived rotational temperature and column density parameters can be used to predict other likely (i.e., large $\mathrm{S} \mu^{2}$ ) glycine lines in the frequency range of the Friedel et al. (2004) survey. Five such glycine transitions were contained within the survey, but none was detected. The $1 \sigma$ integrated intensity upper limits are given in column 6 of Table 6 . The integrated intensity upper limits are the product of the rms noise level in units of $\mathrm{Jy}_{\mathrm{beam}}{ }^{-1}$ and an assumed linewidth of $12.7 \mathrm{~km} \mathrm{~s}^{-1}$ (the largest linewidth reported by Kuan et al. 2003). Predictions for the minimum expected intensity of these 5 lines are included in footnotes to col. 6 and range from 9 to $18 \sigma$. These missing lines demonstrate that the Figure 6 rotational diagram purported to be glycine is based on incorrectly identified and misassigned lines.

We have produced a rotational temperature diagram that looks reasonable and gives reasonable results for unidentified lines assumed to be glycine, but we have also demonstrated that these rotational temperature diagram results have no power to predict the intensities of other likely glycine lines. We conclude that this particular rotational diagram is the result of misassigned $\mathrm{U}$ lines and cannot be used as reliable independent evidence to support the interstellar molecular identification of glycine. We note that equation A4 or A5 will be dominated by the frequency parameter and, as a result, one can almost always construct a reasonable looking rotational temperature diagram from any forest of weak lines spread across a large enough range in energy of a complicated asymmetric rotor molecule like glycine.

\section{CONCLUSIONS}

As we discussed in $\S 1$, there are several pecularities if the lines reported by Kuan et al. (2003) in Sgr B2 (N-LMH), Orion KL, and W51el/e2 belong to interstellar glycine. Therefore, we examined their assignments of key glycine transitions to the spectral lines listed in Table 1. We noted that their lack of correction for beam dilution amounted to the tacit assumption that their glycine sources have an extended source size with $\Theta_{s} \geq 45$ ". In Orion KL, we found that none of the 15 glycine line assignments could be verified because the reported intensities of all 15 lines predict that a major glycine quartet $\left(\mathrm{J}=19-18, \mathrm{~K}_{-1}\right.$ $=0$ or 1 ) should have been detected in our Orion data from the NRAO $12 \mathrm{~m}$ telescope

\footnotetext{
${ }^{11}$ Kuan et al. (2003) constructed rotation temperature diagrams based on fits that were not weighted by the uncertainties in the integrated intensities.
} 
(Figures 1 and 2). So we can conclude that the Orion spectral lines reported by Kuan et al. (2003) (column 8, Table 1) are not glycine. Previously, the VLA data of Hollis et al. (2003b) established upper limits in Orion for compact glycine sources. Therefore, glycine has not been verified in either extended or in compact sources in Orion. In W51 el/e2, 16 lines were assigned to glycine. Fifteen of the 16 line intensities lend to the predictions that the $\mathrm{J}=19-18, \mathrm{~K}_{-1}=0$ or 1 glycine quartets should have been detected in our W51 data from the NRAO $12 \mathrm{~m}$ telescope (Figures 3 and 4). Since our spectra show a negative result, we can conclude that the reported W51 spectral lines are also not glycine (column 9, Table 1). In the direction of Sgr B2(N-LMH), only 13 lines were assigned to glycine but the situation is slightly more complicated by the high spectral line density. However, it can be simplified by recognizing that, based on both line strength values and intensity calculations, the two strongest glycine lines should be those assigned to lines 26 and 27 in column 7 of Table 1. Line 26 appears with a peak intensity of $300 \mathrm{mK}$ in the Sgr B2(N) band scan data of Nummelin et al. (1998), but the important connected lines, $J=42-41$ and 41-40 in the $\mathrm{K}_{-1}$ $=\mathrm{l}$ or 2 series, are missing altogether (Table 3). Furthermore, line 27 should be stronger than line 26, but it too is missing, along with the connected $J=43-42$ and $42-41$ lines in the $\mathrm{K}_{-1}=0$ or 1 series (Table 4 ). All of these key glycine lines strongly connect major $\mathrm{K}_{-1}$ $=0,1$, or 2 rotational levels, so their absence is not possible if the other Sgr B2(N-LMH) glycine lines are assigned correctly. Therefore, we conclude that the Sgr B2(N-LMH) spectral lines reported by Kuan et al. (2003) (column 7, Table 1) are not glycine. In addition, the VLA data of Hollis et al. (2003a) established upper limits for compact glycine sources in Sgr B2(N-LM). Consequently, glycine has not been verified in Sgr B2(N-LMH) either in extended or in compact sources.

If the 27 lines reported by Kuan et al. (2003) in Orion KL, W51el/e2, and Sgr B2(N$\mathrm{LMH}$ ) are not glycine, what are they? Lines 21, 24, and 26 in Table 1 are the 3 lines reported to be common to all 3 sources. We have argued that vibrationally excited ethyl cyanide $\left(\mathrm{CH}_{3} \mathrm{CH}_{2} \mathrm{CN}\right)$ is a logical assignment for line 21 , and vibrationally excited vinyl cyanide $\left(\mathrm{CH}_{2} \mathrm{CHCN}\right)$ for line 24 . The intensity of line 26 increases as the observational beam width narrows, so it could also be a constituent of a somewhat compact source. Four other reported glycine lines could be possibly assigned to more common species listed in Table 5. Beyond this, it is difficult to comment on other possible line carrier candidates because Kuan et al. (2003) only published sample spectra for 11 of their 27 lines. Finally, we have demonstrated that rotational temperature diagrams can not by themselves be used to identify interstellar molecules.

We are indebted to A. Nummelin for providing data from Sgr B2(N). We thank E. 
B. Churchwell, E. C. Sutton, and W. D. Watson for helpful assistance. We appreciate the useful comments from two referees. J. M. H. gratefully acknowledges research support from H. A. Thronson, Technology Director of the NASA Office of Space Science. We acknowledge support from the Laboratory for Astronomical Imaging at the University of Illinois, and NSF grants AST 99-81363 and AST 02-28953. The laboratory measurements of the glycine spectrum were supported by STCU under contract No. 2132.

\section{APPENDIX A}

\section{SOME COMMENTS ABOUT ROTATIONAL TEMPERATURE DIAGRAMS}

The conventional method for forming a rotational temperature diagram is to plot the logarithm of normalized column density vs. upper state rotational energy level. However, this approach incorporates several assumptions. First, it is assumed that each region in the interstellar cloud has uniform physical conditions and that the populations of the energy levels can be characterized by a Boltzmann distribution. Second, optically thin conditions are assumed but it may be possible to correct for this assumption later in the analysis. Third, if it is not possible to measure the source size, it must be assumed the source fills the observational beam. So, if the population distribution can be described adequately by a single rotational temperature and if all the lines are optically thin, the measured fluxes are proportional to the column densities in the upper levels of the transitions being observed.

For autocorrelation (single-element telescope) observations, we can start with equation (1) in $\S 3$. For cross-correlation (interferometric array) observations, we can use (see, for example, Snyder et al. 2001)

$$
<N_{T}>=\frac{2.04 W_{\mathrm{I}} Z e^{E_{\mathrm{u}} / T_{\mathrm{rot}}}}{B \theta_{\mathrm{a}} \theta_{\mathrm{b}} S \mu^{2} \nu^{3}} \times 10^{20} \mathrm{~cm}^{-2} .
$$

In equation (A1), $W_{\mathrm{I}}=\int I_{\nu} d v$ in Jy beam ${ }^{-1} \mathrm{~km} \mathrm{~s}^{-1}$, and $I_{\nu}$ is the flux density per beam. $Z$ is the rotational partition function, B is the beam filling factor, $\theta_{\mathrm{a}}$ and $\theta_{\mathrm{b}}$ are the FWHM synthesized beam dimensions in $\operatorname{arcsec}, \mathrm{S}$ is the linestrength, $\mu^{2}$ the square of the dipole moment in Debye $^{2}$, and $\nu$ is the frequency in GHz. The beam-averaged upper level column density, $\left\langle N_{u}\right\rangle$, is defined by

$$
\frac{<N_{u}>}{g_{u}}=\frac{<N_{T}>}{Z} e^{-\frac{E_{u}}{T_{\text {rot }}}},
$$

where $\mathrm{g}_{u}$ is the statistical weight of the upper level $(2 \mathrm{~J}+1)$. A standard approach is to take the Naperian (base e) logarithm of equation (A2), 


$$
\ln \left(\frac{<N_{u}>}{g_{u}}\right)=-\frac{E_{u}}{T_{\text {rot }}}+\ln \left(\frac{<N_{T}>}{Z}\right) .
$$

Then for cross-correlation data, equations (A1) and (A3) give

$$
\ln \left(\frac{<N_{u}>}{g_{u}}\right)=\ln \left(\frac{2.04 W_{\mathrm{I}} 10^{20}}{B \theta_{\mathrm{a}} \theta_{\mathrm{b}} S \mu^{2} \nu^{3}}\right)=-\frac{E_{u}}{T_{r o t}}+\ln \left(\frac{<N_{T}>}{Z}\right) .
$$

A linear least-squares fit to equation (A4) can be used to construct a rotational temperature diagram where the slope gives the negative reciprocal of the rotational temperature. This temperature can be used to estimate the total molecular column density $\left\langle N_{T}\right\rangle$. The rotational temperature will be equal to the kinetic temperature only at sufficiently high densities. A similar approach can be followed with equations (1) and (A2) to construct a rotational temperature diagram from autocorrelation (single-element telescope) data:

$$
\ln \left(\frac{<N_{u}>}{g_{u}}\right)=\ln \left(\frac{1.67 W_{\mathrm{T}} 10^{14}}{S \mu^{2} \nu}\right)=-\frac{E_{u}}{T_{r o t}}+\ln \left(\frac{<N_{T}>}{Z}\right) .
$$

There are two more important caveats about rotational temperature diagrams that must be noted. First, if common (Briggsian) base 10 logarithms are used, the result is

$$
\log _{10}\left(\frac{<N_{u}>}{g_{u}}\right)=-\frac{E_{u} \log _{10} e}{T_{\text {rot }}}+\log _{10}\left(\frac{<N_{T}>}{Z}\right)
$$

This approach slightly complicates the rotational temperature term. However, it makes the data scatter appear to be smaller since $\log _{10} \frac{\left\langle N_{u}\right\rangle}{g_{u}}=0.434 \ln _{e} \frac{\left\langle N_{u}\right\rangle}{g_{u}}$. The second important caveat is in either equations (A4) or equation (A5), the frequency parameter will dominate the integrated intensity, line strength, and dipole moment terms for typical spectral line data in the millimeter or submillimeter range. Hence, rotational temperature diagrams can not be used as independent tools to verify interstellar spectral line identifications. This point is illustrated in $\S 5$.

If the emission is not optically thin, the finite opacity produces an underestimate in the upper level column density of the observed transition and it can also produce an error in the rotational temperature. Based on the discussion presented by Goldsmith \& Langer (1999), the optical depth, $\tau$, can be used to define an optical depth correction factor, $C_{\tau}$, where

$$
C_{\tau}=\frac{\tau}{1-e^{-\tau}}=\frac{<N_{\mathrm{u}}>}{<N_{\mathrm{u}}^{\text {thin }}>}
$$


From equations (A1) and (A2), we find for cross-correlation data

$$
<N_{\mathrm{u}}^{\text {thin }}>=\left(\frac{2.04 g_{\mathrm{u}} W_{\mathrm{I}} \times 10^{20}}{B \theta_{\mathrm{a}} \theta_{\mathrm{b}} S \mu^{2} \nu^{3}}\right) \mathrm{cm}^{-2} .
$$

Since $C_{\tau} \geq 1$, it has been described by Goldsmith \& Langer (1999) as the factor by which $<N_{\mathrm{u}}^{\text {thin }}>$ (derived directly from $W_{\mathrm{I}}$ ) can appear to be too small because of a finite optical depth. Note that $C_{\tau}$ can be different for each independent transition because $\tau$ is proportional to $S \mu^{2}$. For finite optical depths, we find

$$
\ln \left(\frac{<N_{\mathrm{u}}^{\text {thin }}>}{g_{\mathrm{u}}}\right)=\ln \left(\frac{2.04 W_{\mathrm{I}} \times 10^{20}}{B \theta_{\mathrm{a}} \theta_{\mathrm{b}} S \mu^{2} \nu^{3}}\right)=\ln \left(\frac{<N_{T}>}{Z}\right)-\ln C_{\tau}-\frac{E_{\mathrm{u}}}{T_{\mathrm{rot}}},
$$

which is analogous to equation (24) of Goldsmith \& Langer (1999). Clearly, $\ln C_{\tau}$ lowers the ordinate intensity from the optically thin value for each point on the rotational intensity diagram. We can't calculate a realistic value of $\ln C_{\tau}$ for glycine as no interstellar glycine line identification has been confirmed. However, Goldsmith \& Langer (1999) modeled $\tau$ values for methanol (an esablished interstellar asymmetric rotor) that ranged from 0.25 to 30 . If $\tau=30$ for a given glycine line, that point on the rotational temperature diagram would be depressed by 3.4, a significant amount. On the other hand, $\tau=1$ would reduce the ordinate intensity by only 0.5 . 


\section{REFERENCES}

Berulis, I. I., Winnewisser, G., Krasnov, V. V., \& Sorochenko, R. L. 1985, Sov. Astron. Lett. 11, 251

Bevington, P. R, \& Robinson D. K. 1992, Data Reduction and Error Analysis for the Physical Sciences (2d ed.; New York: McGraw-Hill), 106

Born, M., \& Wolf, E. 1980, Electromagnetic Theory of Propagation Interference and Diffraction of Light (6th ed.; New York: Pergamon Press), 397

Brown, R. D., Godfrey, P. D., Storey, J. V. W., Bassez, M.-P., Robinson, B. J., Batchelor, R. A., McColloch, M. G., Rydbeck, O. E. H., \& Hjalmarson, A. G., 1979, MNRAS, 186, 5P Ceccarelli, C., Loinard, L., Castets, A., Faure, A., \& Lefloch, B. 2000, A\&A, 362, 1122

Combes, F., Nguyen-Q-Rieu, \& Wlodarczak, G. 1996, A\&A, 308, 618

Dickens, J. E., Irvine, W. M., Ohishi, M., Ikeda, M., Ishikawa, S., Nummelin, A., \& Hjalmarson, Å. 1997, ApJ, 489, 753

Friedel, D. N., Snyder, L. E., Turner, B. E., \& Remijan, A. 2004, ApJ, 600, 234

Goldsmith, P. F., \& Langer, W. D. 1999, ApJ, 517, 209

Groner, P., Albert, S., Herbst, E., DeLucia, F. C., Lovas, F. J., Drouin, B. J., \& Pearson, J. C. 2002, ApJS, 142, 145

Guelin, M., \& Chernicharo, J. 1989, in The Physics and Chemistry of Interstellar Molecules, ed. G. Winnewisser \& T. Armstrong, (Berlin: Springer-Verlag), 337

Hollis, J. M., Pedelty, J. A., Boboltz, D. A., Liu, S.-Y., Snyder, L. E., Palmer, P., Lovas, F. J.,\& Jewell, P. R. 2003a, ApJ, 596, L235

Hollis, J. M., Pedelty, J. A., Snyder, L. E., Jewell, P. R., Lovas, F. J., Palmer, P., \& Liu, S.-Y. 2003b, ApJ, 588, 353

Hollis, J. M., Snyder, L. E., Suenram, R. D., \& Lovas, F. J., 1980, ApJ, 241, 1001

Ilyushin, V.V., Alekseev, E.A., Dyubko, S.F, Podnos, S.V, Kleiner, I, Margules, L., Wlodarczak, G. Demaison, J., Cosleou, J., Mate, B., Karyakin, E. N., Golubiatnikov, G. Yu., Fraser, G.T., Suenram, R. D., \& Hougen, J. T. 2001 J. Mol. Spectrosc. 205, 286

Jewell, P. R. 1990, Users Manual for the NRAO 12 m Millimeter-Wave Telescope, National Radio Astronomy Observatory publication

Jenkins, F. A., \& White, H. E. 1957, Fundamentals of Optics (New York: McGraw-Hill)

Kaushik, V. K., Takagi, K., \& Matsumura, C. 1980, J. Mol. Spectrosc. 82, 418

Kuan, Y.-J, Charnley, S. B., Huang, H.- C., Tseng, W.-L., \& Kisiel, Z. 2003, ApJ, 593, 848 
Kutner, M. L., \& Ulich, B. L. 1981, ApJ, 250, 341

Lee, C. W., \& Cho, S.-H. 2002, J. Korean Astron. Soc. 35, 187

Liu, S.-Y., Girart, J. M., Remijan, A., \& Snyder, L. E. 2002, ApJ, 576, 255

Liu, S.-Y., Mehringer, D. M., \& Snyder, L. E. 2001, ApJ, 552, 654

Liu, S.-Y., \& Snyder, L. E. 1999, ApJ, 523, 683

Lovas, F. J. 2004, J Phys. Chem. Ref. Data 33, 177

Lovas, F.J., Kawashima, Y., Grabow, J.-U., Suenram, R.D., Fraser, G.T., \& Hirota, E. 1995, ApJ, 455, L201

Mehringer, D.M., Pearson, J.C., Keene, J., \& Phillips, T.G. 2004, ApJ, 608, 306

Nummelin, A., \& Bergman, P. 1999, A\&A, 341, L59

Nummelin, A., Bergman, P., Hjalmarson,Å., Friberg, P., Irvine, W.M., Millar, T.J., Ohishi, M., \& Saito, S. 1998, ApJS, 117, 427.

Mehringer, D.M., Pearson, J.C., Keene, J., \& Phillips, T.G. 2004, ApJ, 608, 306

Nummelin, A., \& Bergman, P. 1999, A\&A, 341, L59

Nummelin, A.,Bergman, P., Hjalmarson,A. , Friberg, P., Irvine, W.M., Millar, T.J., Ohishi, M., \& Saito, S. 1998, ApJS, 117, 427.

Pickett, H. M., Poynter, R. L., Cohen, E. A., Delitsky, M. L., Pearson, J. C., \& Muller, H. S. P. 1998, J. Quant. Spectrosc. \& Rad. Transfer 60, 883

Reid, M. J., \& Moran, J. M. 1988, in Galactic and Extragalactic Radio Astronomy, ed. G. L. Verschuur \& K. I. Kellerman, (Berlin:Springer-Verlag), 255

Remijan, A., Snyder, L. E., Friedel, D. N., Liu, S.-Y., \& Shah, R. Y. 2003, ApJ, 590, 314

Remijan, A., Snyder, L. E., Liu, S.-Y., Mehringer, D., \& Kuan, Y.-J. 2002, ApJ, 576, 264

Rohlfs, K. \& Wilson, T. L. 2000, Tools of Radio Astronomy (3rd ed.; Berlin: Springer), 141

Salter, C., Maddalena, R. J., \& Garwood, B. 1995, The UniPOPS Cookbook (Greenbank:NRAO)

Snyder, L. E. 1997, Orig. Life \& Evol. Biosphere, 27, 115

Snyder, L. E., Hollis, J. M., Suenram, R. D., Lovas, F. J., Brown, L. W., \& Buhl, D., 1983, ApJ, 268, 123

Snyder, L. E., Lovas, F. J., Mehringer, D. M., Miao, N. Y., Kuan, Y.-J., Hollis, J. M., \& Jewell, P. R. 2002, ApJ, 578, 245

Snyder, L. E., Veal, J. M., Woodney, L. M., Wright, M. C. H., Palmer, P., A'Hearn, M. F., Kuan, Y.-J., de Pater, I, \& Forster, J. R. 2001, AJ, 121, 1147 
Sommerfeld, A. 1964, Optics (New York: Academic Press), 290

Suenram, R.D. \& Lovas, F.J. 1980, J. Am. Chem. Soc. 102, 7180.

Taylor, B.N. \& Kuyatt, C.E. 1994, NIST Tech. Note 1297, U.S. Govt. Printing Office, Washington, DC 20402

Townes, C. H., \& Schawlow, A. L. 1975, Microwave Spectroscopy (New York: Dover), 94

Ulich, B. L., \& Haas, R. W. 1975, ApJS, 30, 247

Watson, J.K.G. 1977, in Vibrational Spectra and Structure. A Series of Advances, Vol. 6, ed. J. R. Durig (New York: Elsevier), 19 


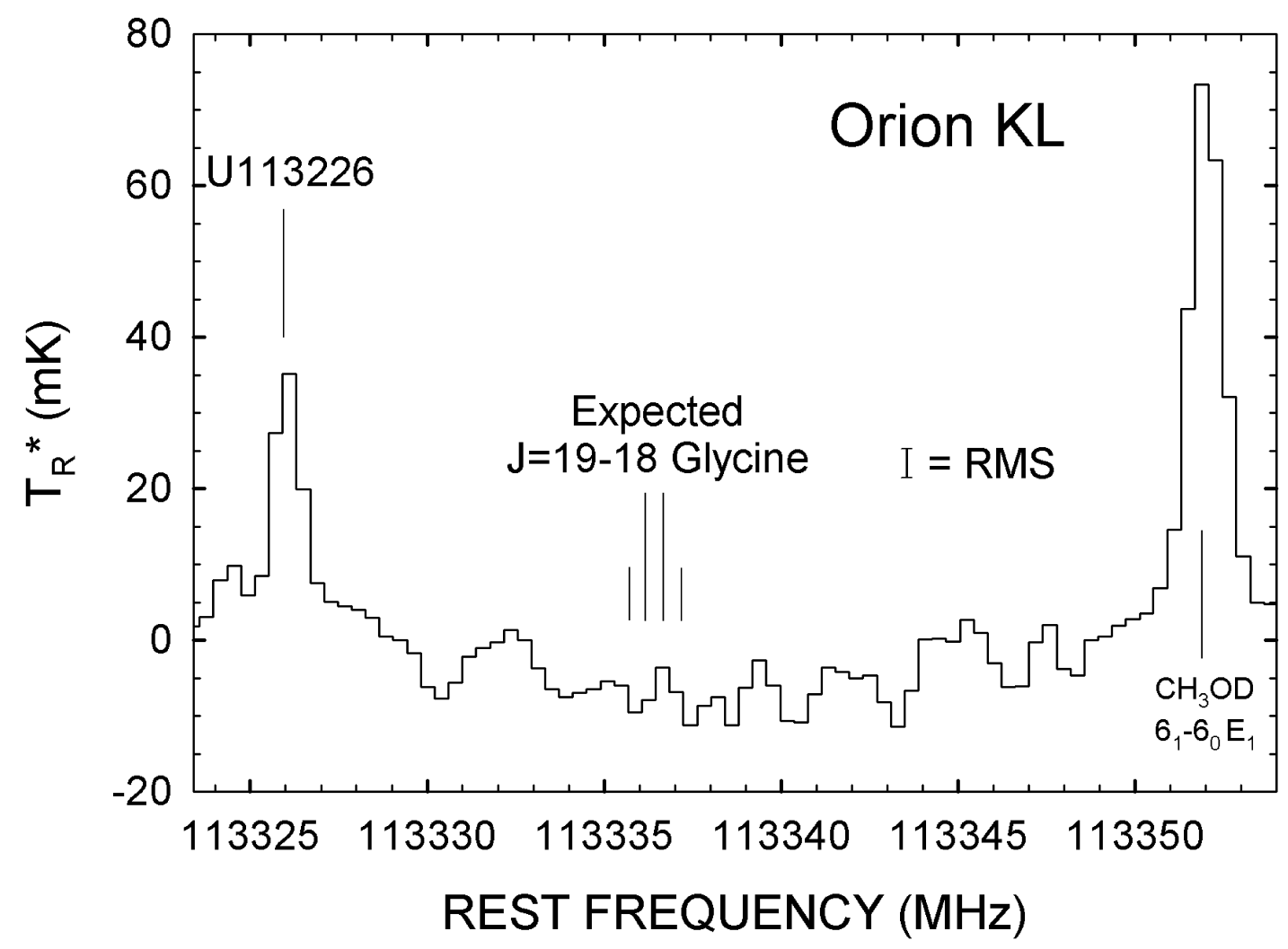

Fig. 1.- Orion KL spectra from 113,323 to 113,355 MHz (centered at 113,339 MHz) observed with the Hybrid Spectrometer on the NRAO $12 \mathrm{~m}$. The spectral positions of the negative results for the nearly four-fold degenerate $\mathrm{J}=19-18$ glycine lines are marked by the four vertical lines centered at 113,336 MHz. U113.226 is on the left, and $\mathrm{CH}_{3} \mathrm{OD}$ is on the right. The ordinate is in units of $\mathrm{mK}$ on the $\mathrm{T}_{R}^{*}$ scale. The abscissa is rest frequency calculated with respect to $\mathrm{V}_{\mathrm{LSR}}=9 \mathrm{~km} \mathrm{~s}^{-1}$ (for the Orion compact ridge) except the $\mathrm{CH}_{3} \mathrm{OD}$ rest frequency is with respect to $\mathrm{V}_{\mathrm{LSR}}=5.6 \mathrm{~km} \mathrm{~s}^{-1}$ (representative of the Orion hot core). The rms noise level for the spectral region between $\mathrm{U} 113226$ and $\mathrm{CH}_{3} \mathrm{OD}$ is $3.7 \mathrm{mK}$.. 


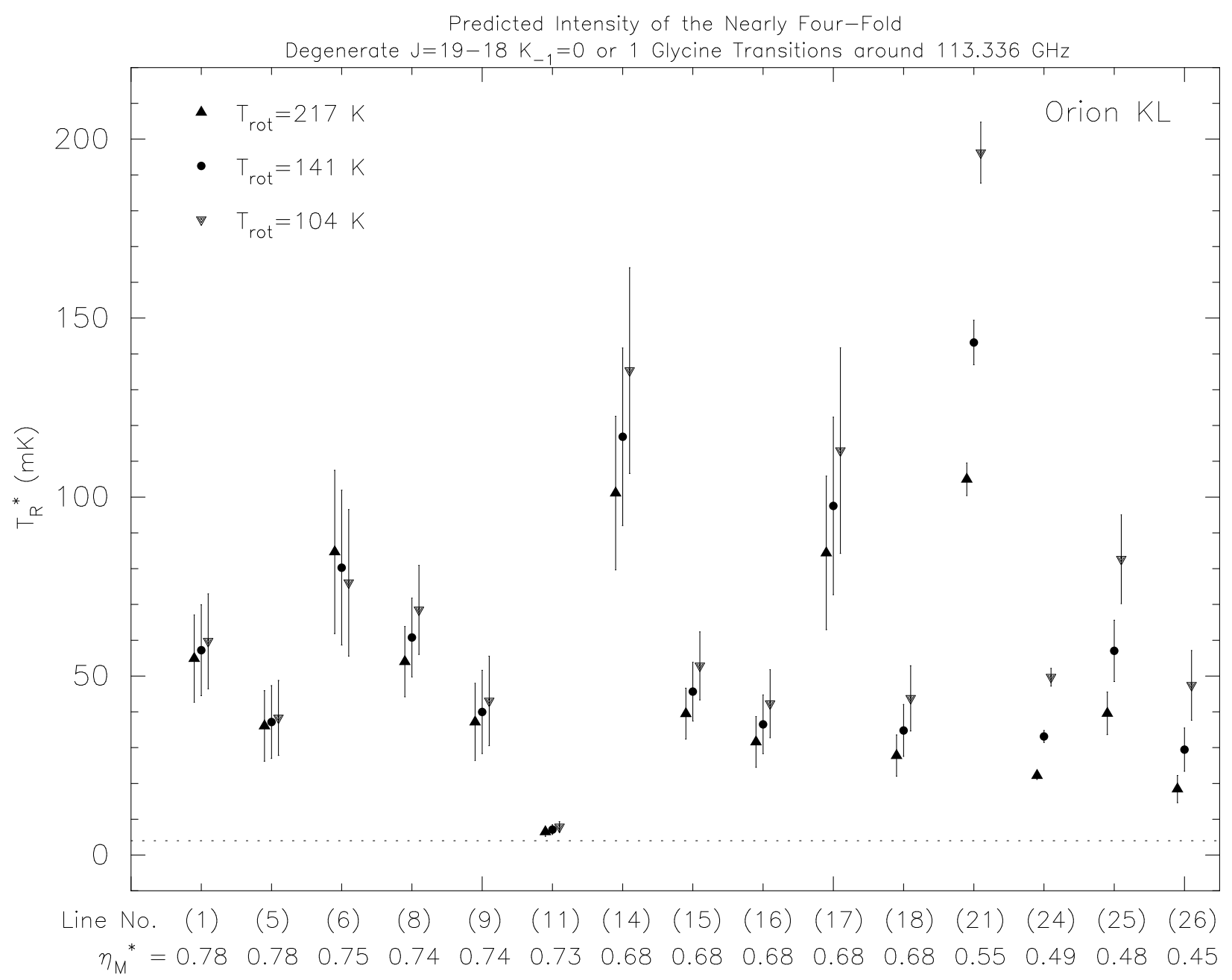

Fig. 2. - The intensity of each of the 15 Orion KL glycine lines reported by Kuan et al. (2003) was used to independently predict the intensity of the nearly four-fold degenerate $\mathrm{J}$ = 19-18 glycine lines at $113.366 \mathrm{GHz}$. A 45" extended source of glycine was assumed. As in Figure 1, the ordinate is in units of $\mathrm{mK}$ based on the $\mathrm{T}_{R}^{*}$ scale. The upper abscissa lists the glycine line number from Table 1, and the lower gives the corresponding main beam efficiency, $\eta_{M}^{*}$, for the NRAO $12 \mathrm{~m}$. Each predicted intensity is calculated for the Orion rotational temperature and its uncertainties, $\mathrm{T}_{\text {rot }}=141_{-37}^{+76} \mathrm{~K}$, derived by Kuan et al. (2003) $(\boldsymbol{\Lambda}=217 \mathrm{~K} ; \bullet=141 \mathrm{~K} ; \boldsymbol{\nabla}=104 \mathrm{~K})$. The uncertainties for each rotational temperature point are based on the rms noise level given for each glycine line reported by Kuan et al. (2003) (see Table 1). The dotted line shows the $3.7 \mathrm{mK}$ noise level from Figure 1. The intensities predicted from all 15 reported glycine lines show that the 19-18 glycine lines should have been detected in Orion KL, but they were not (see Figure 1). 


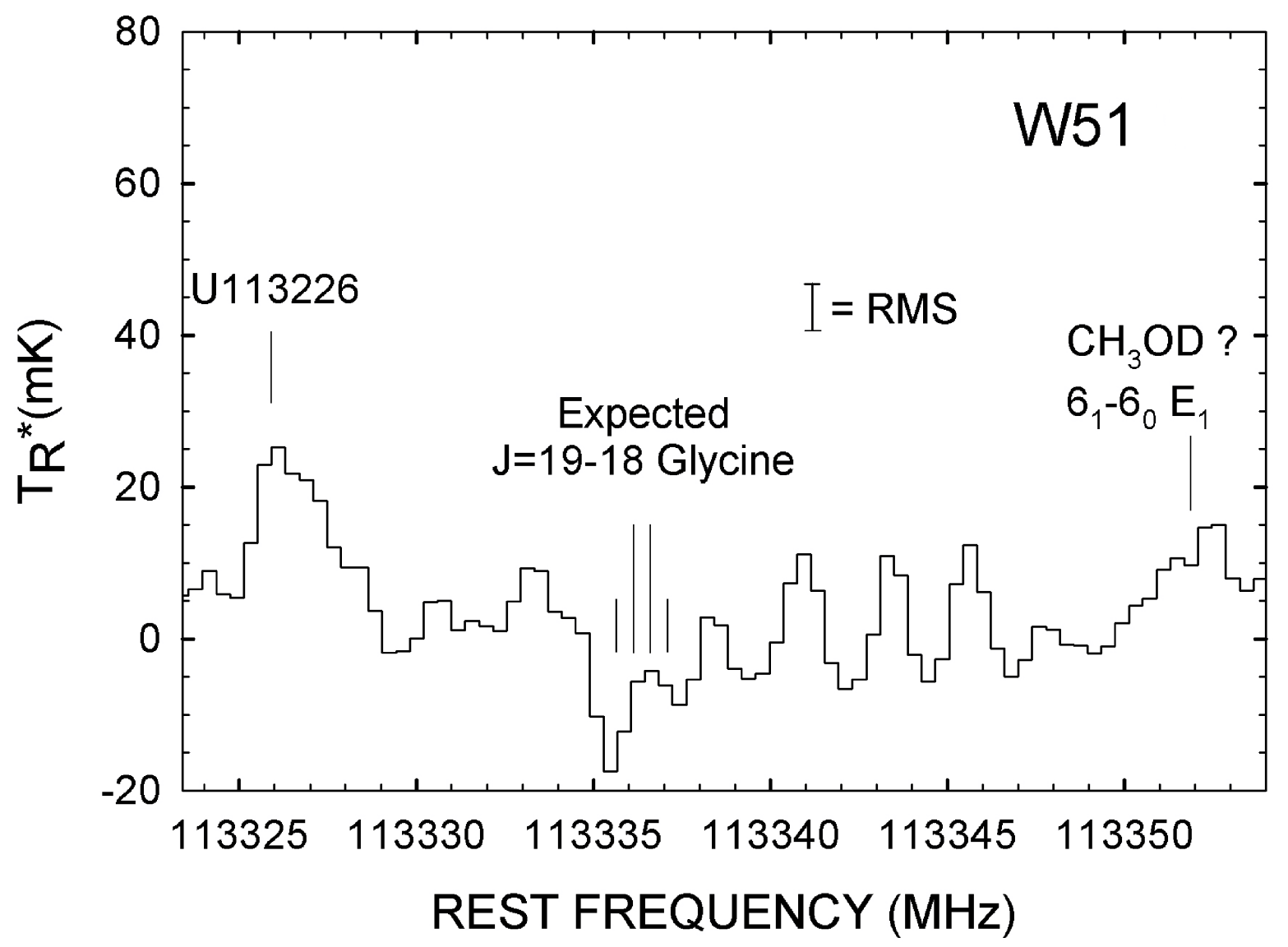

Fig. 3.- W51 spectra from 113,323 to $113,355 \mathrm{MHz}$ (centered at 113,339 MHz) observed with the Hybrid Spectrometer on the NRAO $12 \mathrm{~m}$. The spectral positions of the negative results for the nearly four-fold degenerate $\mathrm{J}=19-18$ glycine lines are marked by the four vertical lines centered at 113,336 MHz. U113.226 is on the left, and a weak emission feature from $\mathrm{CH}_{3} \mathrm{OD}$ is on the right. As in Figure 1, the ordinate is in units of $\mathrm{mK}$ on the $\mathrm{T}_{R}^{*}$ scale. The abscissa is rest frequency calculated with respect to $\mathrm{V}_{\mathrm{LSR}}=57.1 \mathrm{~km} \mathrm{~s}^{-1}$. The rms noise level for the spectral region between $\mathrm{U} 113226$ and $\mathrm{CH}_{3} \mathrm{OD}$ is $6.4 \mathrm{mK}$. 


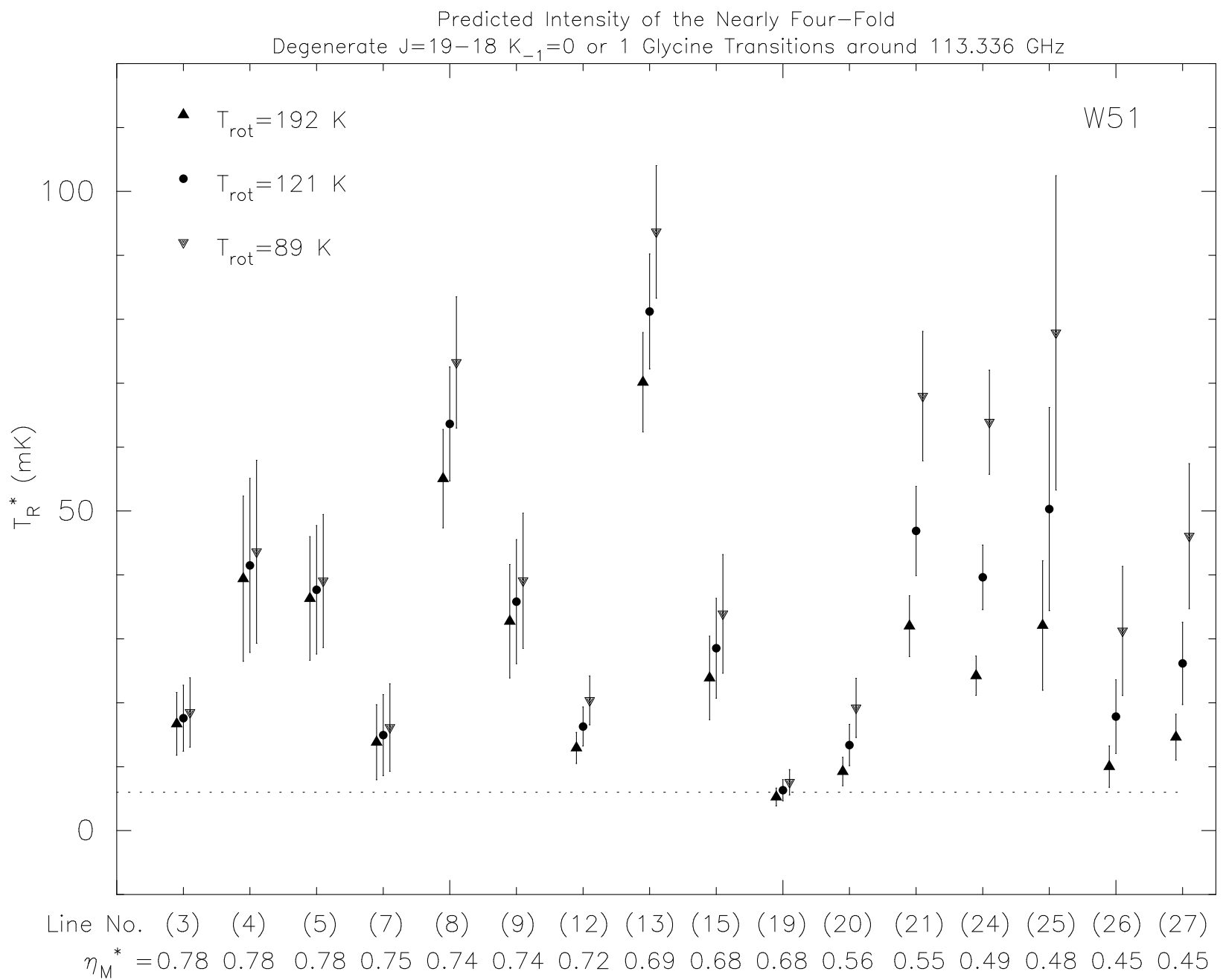

Fig. 4.- The intensity of each of the 16 W51 glycine lines reported by Kuan et al. (2003) was used to independently predict the intensity of the nearly four-fold degenerate $\mathrm{J}=19$-18 glycine lines at $113.366 \mathrm{GHz}$. A 45" extended source of glycine was assumed. As in Figure 1, the ordinate is in units of $\mathrm{mK}$ based on the $\mathrm{T}_{R}^{*}$ scale. The upper abscissa lists the glycine line number from Table 1 , and the lower gives the corresponding main beam efficiency, $\eta_{M}^{*}$, for the NRAO $12 \mathrm{~m}$. Each predicted intensity is calculated for the W51 rotational temperature and its uncertainties, $\mathrm{T}_{\text {rot }}=121_{-32}^{+71} \mathrm{~K}$, derived by Kuan et al. $(2003)(\boldsymbol{\Lambda}=192 \mathrm{~K} ; \bullet=121$ $\mathrm{K} ; \boldsymbol{\nabla}=89 \mathrm{~K})$. The uncertainties for each rotational temperature point are based on the rms noise level given for each glycine line reported by Kuan et al. (2003) (see Table 1). The dotted line shows the $6.4 \mathrm{mK}$ noise level from Figure 3. The intensities predicted from 15 of the 16 reported glycine lines show that the 19-18 glycine lines should have been detected in W51, but they were not (see Figure 3). 

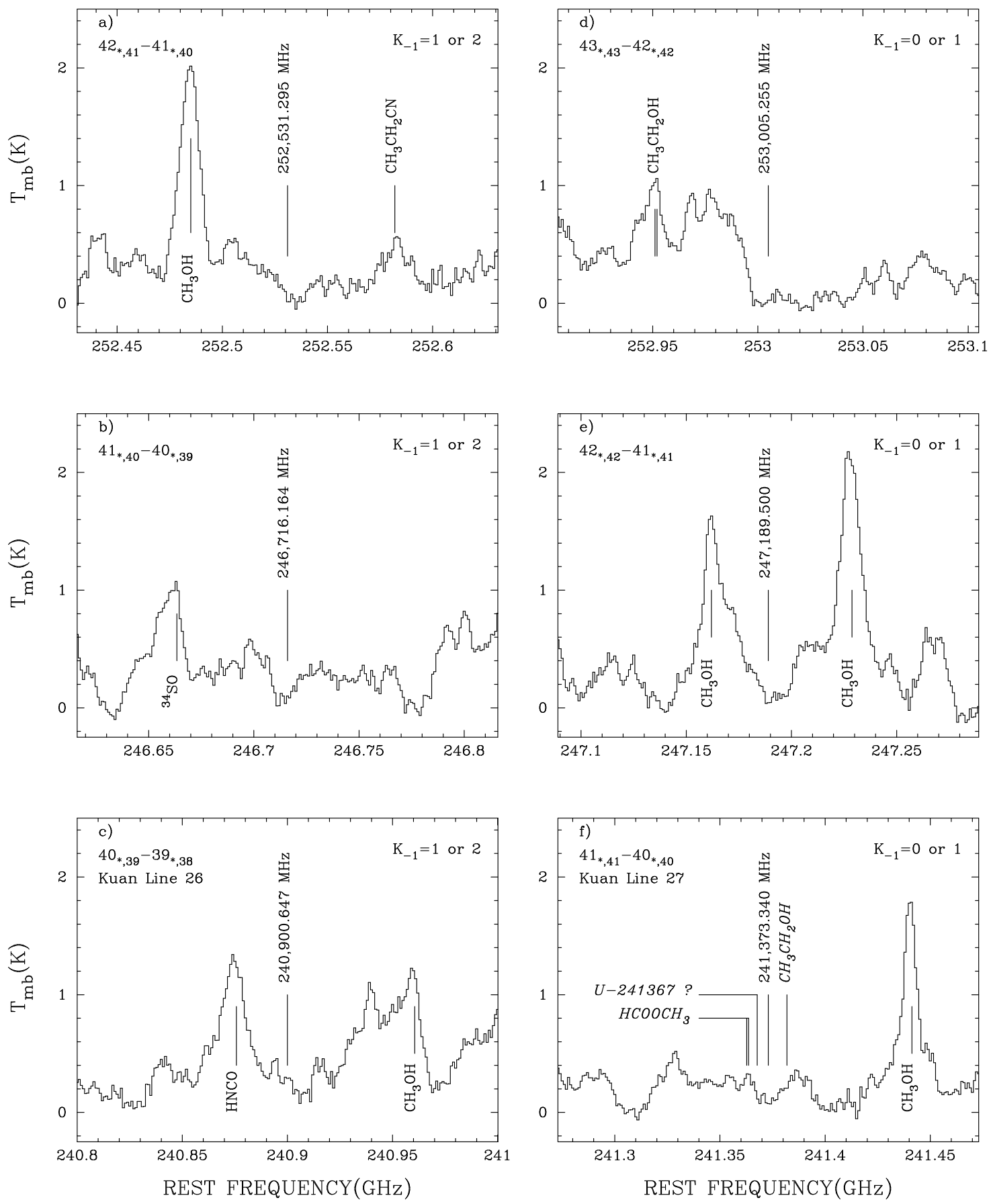

Fig. 5.- This figure is adapted from the Sgr B2(N) data and molecular assignments given by Nummelin et al. (1998). (a) The Nummelin et al. (1998) data for the missing glycine lines for the $\mathrm{K}_{-1}=1$ or 2 frequencies of the fourfold degenerate glycine quartet $\mathrm{J}=42$ 41 at $252,531.295 \mathrm{MHz}$. (b) The missing fourfold degenerate glycine quartet $\mathrm{J}=41$ - 40 at 246,716.164 MHz. (c) The data for line 26 of Kuan et al. (2003), which is reported to be the fourfold degenerate glycine quartet $\mathrm{J}=40$ - 39 at 240,900.647 MHz. (d) The data for the missing glycine lines for the $\mathrm{K}_{-1}=0$ or 1 frequencies of the fourfold degenerate glycine quartet $\mathrm{J}=43$ - 42 at $253,005.255 \mathrm{MHz}$. (e) The missing fourfold degenerate glycine quartet $\mathrm{J}=42$ - 41 at $247,189.500 \mathrm{MHz}$. (f) The missing fourfold degenerate glycine quartet $\mathrm{J}=41$ - 40 at $241,373.340 \mathrm{MHz}$ (line 27 of Kuan et al. 2003). 


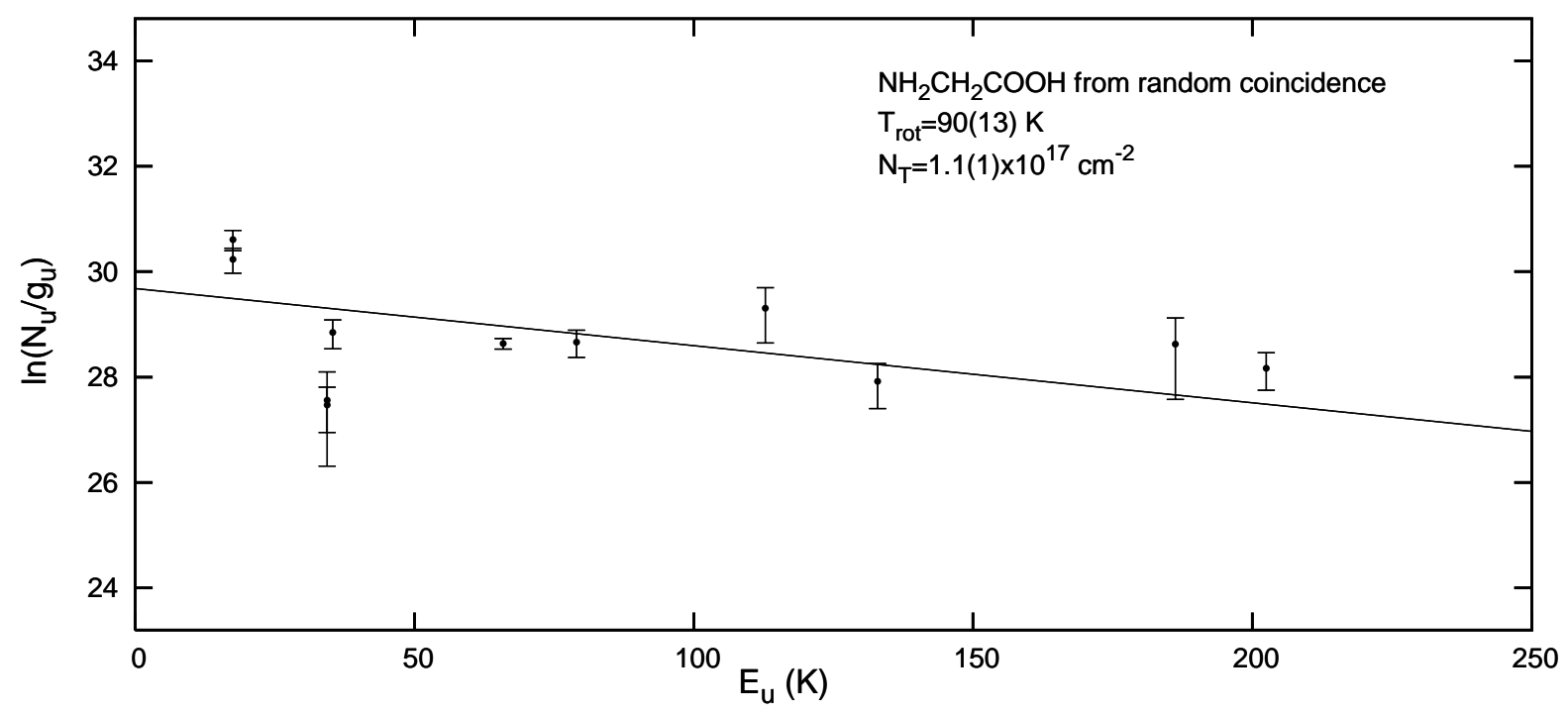

Fig. 6.- Pseudo glycine rotational temperature diagram based on U lines incorrectly assigned to glycine transitions. Table $6 \mathrm{U}$ line data (Friedel et al. 2004) were taken with the BIMA array toward SgrB2(N-LMH) and are approximately frequency coincident with glycine transitions. The ordinate is the Naperian logarithm of the beam-averaged upper level column density, $\left\langle N_{u}\right\rangle$, divided by $\mathrm{g}_{u}$, the statistical weight of the upper level $(2 \mathrm{~J}+1)$; the abscissa $E_{u}$ is the upper state rotational energy level for each transition. As discussed in the text, this diagram may appear to be reasonable and yield a rotation temperature in a range characteristic of the source, but it has no power to predict intensities of glycine lines as shown in Table 6 . Such a diagram cannot be used as evidence of interstellar glycine (see text). 
Table 1. Summary of Reported Glycine Line Detections

\begin{tabular}{|c|c|c|c|c|c|c|c|c|c|c|c|}
\hline \multirow[b]{2}{*}{$\begin{array}{l}\text { Line } \\
\text { No. }\end{array}$} & \multirow[b]{2}{*}{ Transition } & \multirow[b]{2}{*}{ Type } & \multirow{2}{*}{$\begin{array}{c}\text { Rest } \\
\text { Frequency } \\
(\mathrm{MHz})^{\mathrm{a}}\end{array}$} & \multirow{2}{*}{$\begin{array}{c}\text { S } \\
\text { Line } \\
\text { Strength }\end{array}$} & \multirow[b]{2}{*}{$\begin{array}{l}\mathrm{E}_{u} \\
(\mathrm{~K})\end{array}$} & \multicolumn{2}{|c|}{ Sgr B2(N-LMH) } & \multicolumn{2}{|c|}{ Orion-KL } & \multicolumn{2}{|c|}{ W51 e1/e2 } \\
\hline & & & & & & $\begin{array}{c}\mathrm{v}_{L S R} \\
\left(\mathrm{~km} \mathrm{~s}^{-1}\right)^{\mathrm{b}}\end{array}$ & $\begin{array}{c}\mathrm{T}_{R}^{*} \\
(\mathrm{mK})^{\mathrm{c}}\end{array}$ & $\begin{array}{c}\mathrm{v}_{L S R} \\
\left(\mathrm{~km} \mathrm{~s}^{-1}\right)^{\mathrm{b}}\end{array}$ & $\begin{array}{c}\mathrm{T}_{R}^{*} \\
(\mathrm{mK})^{\mathrm{c}}\end{array}$ & $\begin{array}{c}\mathrm{v}_{L S R} \\
\left(\mathrm{~km} \mathrm{~s}^{-1}\right)^{\mathrm{b}}\end{array}$ & $\begin{array}{c}\mathrm{T}_{R}^{*} \\
(\mathrm{mK})^{\mathrm{c}}\end{array}$ \\
\hline 1 & $21_{1,20}-20_{2,19}$ & $\mathrm{~b}$ & $130346.775(13)$ & 16.7 & 72.3 & $66.3(1.2)$ & $26.0(5.9)$ & $8.0(3.1)$ & $9.0(2.0)$ & \multicolumn{2}{|c|}{ U Interloper ${ }^{\mathrm{d}}$} \\
\hline 2 & $21_{2,20}-20_{2,19}$ & $\mathrm{a}$ & $130354.371(13)$ & 20.5 & 72.3 & $66.3(1.2)$ & $30.0(5.9)$ & \multicolumn{2}{|c|}{ U Interloper } & \multicolumn{2}{|c|}{ U Interloper } \\
\hline 3 & $21_{1,20}-20_{1,19}$ & $\mathrm{a}$ & $130360.890(13)$ & 20.5 & 72.3 & \multicolumn{2}{|c|}{ U Interloper } & U Inte & rloper & $60.0(3.1)$ & $7.5(2.2)$ \\
\hline 4 & $21_{2,20}-20_{1,19}$ & $\mathrm{~b}$ & $130368.485(13)$ & 16.7 & 72.3 & ID-Inte & rloper ${ }^{\mathrm{e}}$ & ID-Int & erloper & $60.0(3.1)$ & $6.4(2.1)$ \\
\hline 5 & $19_{3,16}-18_{3,15}$ & $\mathrm{a}$ & $131423.627(13)$ & 18.1 & 67.6 & Not $\mathrm{Ob}$ & served $^{f}$ & $8.5(1.1)$ & $15.0(4.1)$ & $60.0(3.0)$ & $15.0(4.0)$ \\
\hline 6 & $12_{5,8}-11_{4,7}$ & $\mathrm{~b}$ & $142225.340(120)$ & 4.6 & 34.0 & $64.0(5.6)$ & $22.0(2.0)$ & $7.8(2.8)$ & $5.2(1.4)$ & Not De & tected $^{g}$ \\
\hline 7 & $21_{3,18}-20_{3,17}$ & $\mathrm{a}$ & $142301.054(13)$ & 20 & 81.0 & $64.0(2.1)$ & $38.0(2.2)$ & ID-Int & erloper & $60.0(2.8)$ & $6.6(2.8)$ \\
\hline 8 & $21_{9,12}-20_{9,11}$ & $\mathrm{a}$ & $144841.736(22)$ & 17.2 & 102.9 & Not $\mathrm{Ob}$ & served & $5.0(2.8)$ & $20.9(3.8)$ & $56.0(2.8)$ & $20.7(2.9)$ \\
\hline 9 & $21_{5,17}-20_{5,16}$ & $\mathrm{a}$ & $144847.191(16)$ & 19.7 & 84.8 & Not $\mathrm{Ob}$ & served & $8.0(1.4)$ & $17.9(5.2)$ & $60.0(1.4)$ & $15.5(4.2)$ \\
\hline 10 & $24_{1,23}-23_{2,22}$ & $\mathrm{~b}$ & $147812.030(17)$ & 19.7 & 92.7 & $64.0(1.0)$ & $20.0(5.0)$ & Not Ol & served & Not $\mathrm{Ob}$ & served \\
\hline & $24_{2,23}-23_{2,22}$ & $\mathrm{a}$ & $147813.168(17)$ & 23.5 & $"$ & & & & & & \\
\hline & $24_{1,23}-23_{1,22}$ & $\mathrm{a}$ & $147814.186(17)$ & $"$ & $"$ & & & & & & \\
\hline & $22_{3,19}-21_{3,18}$ & $\mathrm{a}$ & $147815.031(14)$ & 21 & 88.0 & & & & & & \\
\hline & $24_{2,23}-23_{1,22}$ & $\mathrm{~b}$ & $147815.324(17)$ & 19.7 & 92.7 & & & & & & \\
\hline 11 & $25_{0,25}-24_{1,24}$ & $\mathrm{~b}$ & $148268.007(18)$ & 23.6 & 94.0 & U Inter & cloper & $5.0(2.0)$ & $11.0(2.1)$ & U Inter & rloper \\
\hline & $25_{1,25}-24_{1,24}$ & $\mathrm{a}$ & $148268.016(18)$ & 24.8 & $"$ & & & & & & \\
\hline & $25_{0,25}-24_{0,24}$ & $\mathrm{a}$ & $148268.024(18)$ & $"$ & $"$ & & & & & & \\
\hline & $25_{1,25}-24_{0,24}$ & $\mathrm{~b}$ & $148268.033(18)$ & 23.6 & $"$ & & & & & & \\
\hline 12 & $\begin{array}{c}22_{12,11}-21_{12,10} \\
22_{12,10}-21_{12,9}\end{array}$ & $\begin{array}{l}\mathrm{a} \\
\mathrm{a}\end{array}$ & $\begin{array}{c}\text { 150909.703(20) } \\
"\end{array}$ & $\begin{array}{c}15.5 \\
\quad "\end{array}$ & $\begin{array}{c}130.8 \\
\quad "\end{array}$ & $68.0(2.7)$ & $18.0(2.5)$ & Not Dete & $\operatorname{ted}(\mathrm{S} / \mathrm{N})^{\mathrm{h}}$ & $58.0(2.0)$ & $8.0(1.5)$ \\
\hline 13 & $23_{6,18}-22_{6,17}$ & $\mathrm{a}$ & $160153.431(30)$ & 21.4 & 103.5 & U Inter & cloper & U Inte & rloper & $60.0(2.5)$ & $37.0(4.1)$ \\
\hline 14 & $26_{2,24}-25_{3,23}$ & $\mathrm{~b}$ & $164851.728(30)$ & 18.8 & 113.7 & Not $\mathrm{Ob}$ & served & $7.5(1.2)$ & $21.2(4.5)$ & U Inter & rloper \\
\hline 15 & $26_{3,24}-25_{3,23}$ & $\mathrm{a}$ & $164862.039(30)$ & 25.2 & 113.7 & Not $\mathrm{Ob}$ & served & $7.5(1.2)$ & $25.0(4.5)$ & $60.0(1.2)$ & $14.6(4.0)$ \\
\hline 16 & $26_{2,24}-25_{2,23}$ & $\mathrm{a}$ & $164870.438(30)$ & 25.2 & 113.7 & Not $\mathrm{Ob}$ & served & $7.5(1.2)$ & $20.0(4.5)$ & Not De & tected \\
\hline 17 & $26_{3,24}-25_{2,23}$ & $\mathrm{~b}$ & $164880.749(30)$ & 18.8 & 113.7 & Not $\mathrm{Ob}$ & served & $7.5(1.2)$ & $17.7(4.5)$ & Not Detec & $\operatorname{ted}(\mathrm{S} / \mathrm{N})$ \\
\hline 18 & $24_{12,13}-23_{12,12}$ & $\mathrm{a}$ & $164886.068(33)$ & 18.0 & 146.4 & Not $\mathrm{Ob}$ & served & $7.5(1.2)$ & $21.6(4.5)$ & Not De & tected \\
\hline & $24_{12,12}-23_{12,11}$ & $\mathrm{a}$ & $164886.071(33)$ & $"$ & $"$ & & & & & & \\
\hline 19 & $27_{1,26}-26_{2,25}$ & $\mathrm{~b}$ & $165270.666(22)$ & 22.7 & 115.7 & $64.8(0.9)$ & $60.0(4.6)$ & Not D & tected & $58.2(2.4)$ & $8.0(2.1)$ \\
\hline & $27_{2,26}-26_{2,25}$ & $\mathrm{a}$ & $165270.829(22)$ & 26.5 & $"$ & & & & & & \\
\hline & $27_{1,26}-26_{1,25}$ & $\mathrm{a}$ & $165270.979(22)$ & $"$ & $"$ & & & & & & \\
\hline & $27_{2,26}-26_{1,25}$ & $\mathrm{~b}$ & $165271.142(22)$ & 22.7 & $"$ & & & & & & \\
\hline 20 & $33_{2,31}-32_{3,30}$ & $\mathrm{~b}$ & $205560.595(67)$ & 25.7 & 176.8 & $67.0(0.7)$ & $95.0(11.9)$ & Not D & tected & $58.6(3.9)$ & $17.0(4.1)$ \\
\hline & $33_{3,31}-32_{3,30}$ & $\mathrm{a}$ & $205560.730(67)$ & 32.2 & $"$ & & & & & & \\
\hline & $33_{2,31}-32_{2,30}$ & $\mathrm{a}$ & $205560.849(67)$ & $"$ & $"$ & & & & & & \\
\hline & $33_{3,31}-32_{2,30}$ & $\mathrm{~b}$ & $205560.984(67)$ & 25.7 & $"$ & & & & & & \\
\hline 21 & $35_{0,35}-34_{1,34}$ & $\mathrm{~b}$ & $206468.453(17)$ & 33.6 & 180.5 & $66.0(1.9)$ & $210.0(8.9)$ & $7.3(0.7)$ & $230.0(10.0)$ & $60.5(0.7)$ & $65.0(9.7)$ \\
\hline & $35_{1,35}-34_{1,34}$ & $\mathrm{a}$ & $"$ & 34.8 & $"$ & & & & & & \\
\hline & $35_{0,35}-34_{0,34}$ & $\mathrm{a}$ & $"$ & $"$ & $"$ & & & & & & \\
\hline & $35_{1,35}-34_{0,34}$ & $\mathrm{~b}$ & $"$ & 33.6 & $"$ & & & & & & \\
\hline 22 & $36_{0,36}-35_{1,35}$ & $\mathrm{~b}$ & $212286.829(18)$ & 34.6 & 190.7 & $66.0(0.7)$ & $58.0(15.6)$ & ID-Int & erloper & ID-Inte & erloper \\
\hline & $36_{1,36}-35_{1,35}$ & $\mathrm{a}$ & $"$ & 35.8 & $"$ & & & & & & \\
\hline & $36_{0,36}-35_{0,35}$ & $\mathrm{a}$ & $"$ & $"$ & $"$ & & & & & & \\
\hline & $36_{1,36}-35_{0,35}$ & $\mathrm{~b}$ & $"$ & 34.6 & $"$ & & & & & & \\
\hline 23 & $36_{2,34}-35_{3,33}$ & $\mathrm{~b}$ & $223004.375(88)$ & 28.9 & 208.2 & $67.3(1.8)$ & $40.0(10.5)$ & Not D & tected & Not $\mathrm{Ob}$ & served \\
\hline & $36_{3,34}-35_{3,33}$ & $\mathrm{a}$ & $223004.395(88)$ & 35.2 & $"$ & & & & & & \\
\hline & $36_{2,34}-35_{2,33}$ & $\mathrm{a}$ & $223004.413(88)$ & $"$ & $"$ & & & & & & \\
\hline & $36_{3,34}-35_{2,33}$ & $\mathrm{~b}$ & $223004.432(88)$ & 28.9 & $"$ & & & & & & \\
\hline 24 & $36_{3,33}-35_{4,32}$ & $\mathrm{~b}$ & $228419.435(104)$ & 25.9 & 216.3 & $65.0(1.3)$ & $70.0(7.6)$ & $4.9(3.5)$ & $40.0(2.0)$ & $57.0(1.8)$ & $40.0(5.1)$ \\
\hline & $36_{4,33}-35_{4,32}$ & $\mathrm{a}$ & $228420.029(104)$ & 34.9 & $"$ & & & & & & \\
\hline & $36_{3,33}-35_{3,32}$ & $\mathrm{a}$ & $228420.526(104)$ & $"$ & $"$ & & & & & & \\
\hline & $36_{4,33}-35_{3,32}$ & $\mathrm{~b}$ & $228421.120(104)$ & 25.9 & $"$ & & & & & & \\
\hline 25 & $33_{6,27}-32_{6,26}$ & $\mathrm{a}$ & $230348.993(64)$ & 31.3 & 202.5 & ID-Inte & rloper & $8.0(3.5)$ & $26.0(3.9)$ & $58.7(3.5)$ & $19.0(6.0)$ \\
\hline 26 & $40_{1,39}-39_{2,38}$ & $\mathrm{~b}$ & $240900.647(76)$ & 35.8 & 244.2 & 63.2(0.6) & $94.0(11.0)$ & $5.0(1.7)$ & $35.0(7.2)$ & $57.0(1.7)$ & $17.0(5.5)$ \\
\hline & $40_{2,39}-39_{2,38}$ & $\mathrm{a}$ & $"$ & 39.5 & $"$ & & & & & & \\
\hline & $40_{1,39}-39_{1,38}$ & $\mathrm{a}$ & $"$ & $"$ & $"$ & & & & & & \\
\hline & $40_{2,39}-39_{1,38}$ & $\mathrm{~b}$ & $"$ & 35.8 & $"$ & & & & & & \\
\hline 27 & $41_{0,41}-40_{1,40}$ & $\mathrm{~b}$ & $241373.340(32)$ & 39.6 & 245.9 & $\mathrm{U} / \mathrm{ID}-\mathrm{In}$ & erloper & Not D & tected & $57.0(1.7)$ & $26.0(6.4)$ \\
\hline & $41_{1,41}-40_{1,40}$ & $\mathrm{a}$ & $"$ & 40.8 & $"$ & & & & & & \\
\hline & $41_{0,41}-40_{0,40}$ & $\mathrm{a}$ & $"$ & $"$ & $"$ & & & & & & \\
\hline & $41_{1,41}-40_{0,40}$ & $\mathrm{~b}$ & $"$ & 39.6 & $"$ & & & & & & \\
\hline
\end{tabular}

${ }^{\mathrm{a}} 2 \sigma$ frequency uncertainty is in parentheses.

$\mathrm{b}_{\text {This is the }} \mathrm{v}_{L S R}$ and uncertainty listed by Kuan et al. (2003).

${ }^{\mathrm{c}}$ This is the $\mathrm{T}_{R}^{*}$ and rms noise level listed by Kuan et al. (2003). 


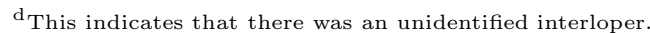

e This indicates that there was an identified interloper.

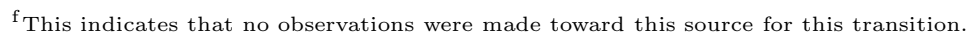

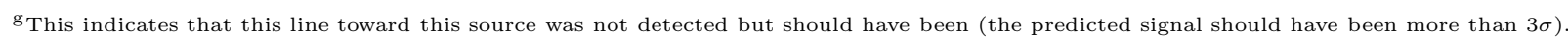

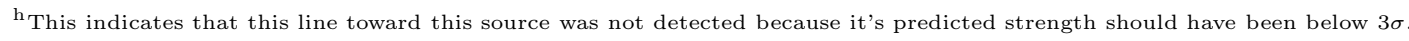


Table 2. $113 \mathrm{GHz}$ Search Frequencies for Orion KL and W51

\begin{tabular}{|c|c|c|c|c|c|c|c|c|c|}
\hline \multirow{2}{*}{$\begin{array}{c}\text { Rest } \\
\text { Frequency } \\
(\mathrm{MHz})^{\mathrm{a}}\end{array}$} & \multirow[b]{2}{*}{ Molecule } & \multirow[b]{2}{*}{ Transition } & \multirow[b]{2}{*}{ Type } & \multirow{2}{*}{$\begin{array}{c}\text { S } \\
\text { Line } \\
\text { Strength }\end{array}$} & \multirow[b]{2}{*}{$\begin{array}{l}\mathrm{E}_{u} \\
(\mathrm{~K})\end{array}$} & \multicolumn{2}{|c|}{ Orion-KL } & \multicolumn{2}{|c|}{ W51 e1/e2 } \\
\hline & & & & & & $\begin{array}{c}\mathrm{T}_{R}^{*} \\
(\mathrm{mK})\end{array}$ & $\begin{array}{c}\Delta \mathrm{v} \\
\left(\mathrm{km} \mathrm{s}^{-1}\right)\end{array}$ & $\begin{array}{c}\mathrm{T}_{R}^{*} \\
(\mathrm{mK})\end{array}$ & $\begin{array}{c}\Delta v \\
\left(\mathrm{~km} \mathrm{~s}^{-1}\right)\end{array}$ \\
\hline 113,326 & U113.326 & $\ldots$ & $\ldots$ & $\ldots$ & $\ldots$ & 36 & 1.6 & 23 & 2.9 \\
\hline $113,335.700(18)$ & $\mathrm{NH}_{2} \mathrm{CH}_{2} \mathrm{COOH}$ & $19_{0,19}-18_{1,18}$ & $\mathrm{~b}$ & 17.6 & 55.5 & $<3.7$ & $\ldots$ & $<6.4$ & $\ldots$ \\
\hline $113,336.209(18)$ & $\mathrm{NH}_{2} \mathrm{CH}_{2} \mathrm{COOH}$ & $19_{1,19}-18_{1,18}$ & $\mathrm{a}$ & 18.8 & 55.5 & $<3.7$ & $\ldots$ & $<6.4$ & $\ldots$ \\
\hline $113,336.696(18)$ & $\mathrm{NH}_{2} \mathrm{CH}_{2} \mathrm{COOH}$ & $19_{0,19}-18_{0,18}$ & $\mathrm{a}$ & 18.8 & 55.5 & $<3.7$ & $\ldots$ & $<6.4$ & $\ldots$ \\
\hline $113,337.205(18)$ & $\mathrm{NH}_{2} \mathrm{CH}_{2} \mathrm{COOH}$ & $19_{1,19}-18_{0,18}$ & b & 17.6 & 55.5 & $<3.7$ & $\ldots$ & $<6.4$ & $\ldots$ \\
\hline $113,250.80(10)^{\mathrm{b}}$ & $\mathrm{CH}_{3} \mathrm{OD}$ & $6_{1}-6_{0} E_{1}$ & $\mathrm{~b}$ & $7.8^{\mathrm{c}}$ & 54.8 & 74 & 1.4 & 11 & 3.6 \\
\hline
\end{tabular}

${ }^{\mathrm{a}} 2 \sigma$ uncertainty is in parentheses.

${ }^{\mathrm{b}}$ Rest Frequency from Kaushik, Takage, \& Matsumura (1980)

${ }^{\mathrm{c}}$ This is the product $\mathrm{S} \mu^{2}$ in Debyes ${ }^{2}$. 
Table 3. Connected $\mathrm{K}_{-1}=1$ or 2 Glycine Transitions in the Nummelin et al. Survey Range

\begin{tabular}{|c|c|c|c|c|c|c|c|}
\hline Transition & Type & $\begin{array}{c}\text { Rest } \\
\text { Frequency } \\
(\mathrm{MHz})^{\mathrm{a}}\end{array}$ & $\begin{array}{c}\text { S } \\
\text { Line } \\
\text { Strength }\end{array}$ & $\begin{array}{l}\mathrm{E}_{u} \\
(\mathrm{~K})\end{array}$ & $\begin{array}{c}\text { Predicted } \\
\mathrm{T}_{m b} \\
(\mathrm{mK})\end{array}$ & $\begin{array}{c}\text { Observed } \\
\mathrm{T}_{m b} \\
(\mathrm{mK})\end{array}$ & Comments \\
\hline $43_{2,42}-42_{2,41}$ & $\mathrm{a}$ & $258,346.029(96)$ & 42.5 & 276.8 & $\cdots$ & $\cdots$ & Masked by $\mathrm{CH}_{3} \mathrm{NH}_{2}$ at $258,349 \mathrm{MHz}^{\mathrm{b}}$. \\
\hline $43_{2,42}-42_{1,41}$ & $\mathrm{~b}$ & $258,346.029(96)$ & 38.8 & 276.8 & & & \\
\hline $43_{1,42}-42_{2,41}$ & $\mathrm{~b}$ & $258,346.029(96)$ & 38.8 & 276.8 & & & \\
\hline $43_{1,42}-42_{1,41}$ & $\mathrm{a}$ & $258,346.029(96)$ & 42.5 & 276.8 & & & \\
\hline $42_{2,41}-41_{2,40}$ & $\mathrm{a}$ & $252,531.295(89)$ & 41.5 & 268.2 & $233_{-55}^{+65}$ & $<100$ & \\
\hline $42_{1,41}-41_{2,40}$ & $\mathrm{~b}$ & $252,531.295(89)$ & 37.8 & 268.2 & & & \\
\hline $42_{2,41}-41_{1,40}$ & $\mathrm{~b}$ & $252,531.295(89)$ & 37.8 & 268.2 & & & \\
\hline $42_{1,41}-41_{1,40}$ & $\mathrm{a}$ & $252,531.295(89)$ & 41.5 & 268.2 & & & \\
\hline $41_{2,40}-40_{2,39}$ & $\mathrm{a}$ & $246,716.164(82)$ & 40.5 & 256.1 & $265_{-53}^{+58}$ & $<100$ & \\
\hline $41_{2,40}-40_{1,39}$ & $\mathrm{~b}$ & $246,716.164(82)$ & 36.8 & 256.1 & & & \\
\hline $41_{1,40}-40_{2,39}$ & $\mathrm{~b}$ & $246,716.164(82)$ & 36.8 & 256.1 & & & \\
\hline $41_{1,40}-40_{1,39}$ & $\mathrm{a}$ & $246,716.164(82)$ & 40.5 & 256.1 & & & \\
\hline $40_{2,39}-39_{2,38}$ & $\mathrm{a}$ & $240,900.647(76)$ & 39.5 & 244.3 & $\mathrm{Std}^{\mathrm{C}}$ & 300 & Line $26^{\mathrm{d}}$. \\
\hline $40_{1,39}-39_{2,38}$ & $\mathrm{~b}$ & $240,900.647(76)$ & 35.8 & 244.3 & & & \\
\hline $40_{2,39}-39_{1,38}$ & $\mathrm{~b}$ & $240,900.647(76)$ & 35.8 & 244.3 & & & \\
\hline $40_{1,39}-39_{1,38}$ & $\mathrm{a}$ & $240,900.647(76)$ & 39.5 & 244.3 & & & \\
\hline $39_{2,38}-38_{2,37}$ & $\mathrm{a}$ & $235,084.758(70)$ & 38.5 & 232.7 & $338_{-68}^{+73}$ & 250 & Line U235085 at $235,085 \mathrm{MHz}^{\mathrm{b}}$ \\
\hline $39_{2,38}-38_{1,37}$ & $\mathrm{~b}$ & $235,084.758(70)$ & 34.8 & 232.7 & & & \\
\hline $39_{1,38}-38_{2,37}$ & $\mathrm{~b}$ & $235,084.758(70)$ & 34.8 & 232.7 & & & \\
\hline $39_{1,38}-38_{1,37}$ & $\mathrm{a}$ & $235,084.758(70)$ & 38.5 & 232.7 & & & \\
\hline $38_{2,37-37_{2,36}}$ & $\mathrm{a}$ & $229,268.511(65)$ & 37.5 & 221.4 & . & $\cdots$ & Masked by $\mathrm{CH}_{3} \mathrm{CH}_{2} \mathrm{CN}$ at $229,262 \mathrm{MHz}^{\mathrm{b}}$ \\
\hline $38_{1,37}-37_{2,36}$ & $\mathrm{~b}$ & $229,268.511(65)$ & 33.8 & 221.4 & & & \\
\hline $38_{2,37}-37_{1,36}$ & $\mathrm{~b}$ & $229,268.511(65)$ & 33.8 & 221.4 & & & \\
\hline $38_{1,37}-37_{1,36}$ & $\mathrm{a}$ & $229,268.511(65)$ & 37.5 & 221.4 & & & \\
\hline $37_{2,36}-36_{2,35}$ & $\mathrm{a}$ & $223,451.918(60)$ & 36.5 & 210.4 & $\cdots$ & $\cdots$ & Masked by $\mathrm{NH}_{2} \mathrm{CHO}$ at $223,449 \mathrm{MHz}^{\mathrm{b}}$ \\
\hline $37_{2,36}-36_{1,35}$ & $\mathrm{~b}$ & $223,451.918(60)$ & 32.8 & 210.4 & & & \\
\hline $37_{1,36}-36_{2,35}$ & $\mathrm{~b}$ & $223,451.918(60)$ & 32.8 & 210.4 & & & \\
\hline $37_{1,36}-36_{1,35}$ & a & $223,451.918(60)$ & 36.5 & 210.4 & & & \\
\hline
\end{tabular}

$\mathrm{a}_{2 \sigma}$ uncertainty is in parentheses.

${ }^{\mathrm{b}}$ Nummelin et al. (1998)

${ }^{c}$ The intensity of this line is the standard on which all other Table 3 and Table 4 predictions are based.

${ }^{\mathrm{d}}$ Kuan et al. (2003) list this as glycine line 26. 
Table 4. Connected $\mathrm{K}_{-1}=0$ or 1 Glycine Transitions in the Nummelin et al. Survey Range

\begin{tabular}{|c|c|c|c|c|c|c|c|}
\hline Transition & Type & $\begin{array}{c}\text { Rest } \\
\text { Frequency } \\
(\mathrm{MHz})^{\mathrm{a}}\end{array}$ & $\begin{array}{c}\mathrm{S} \\
\text { Line } \\
\text { Strength }\end{array}$ & $\begin{array}{c}\mathrm{E}_{u} \\
(\mathrm{~K})\end{array}$ & $\begin{array}{c}\text { Predicted } \\
\mathrm{T}_{m b} \\
(\mathrm{mK})\end{array}$ & $\begin{array}{c}\text { Observed } \\
\mathrm{T}_{m b} \\
(\mathrm{mK})\end{array}$ & Comments \\
\hline $44_{1,44}-43_{1,43}$ & $\mathrm{a}$ & $258,820.597(46)$ & 43.8 & 282.3 & $210_{-57}^{+72}$ & $<200$ & Dominated by narrow noise spikes. ${ }^{b}$ \\
\hline $44_{1,44}-43_{0,43}$ & $\mathrm{~b}$ & $258,820.597(46)$ & 42.6 & 282.3 & & & \\
\hline $44_{0,44}-43_{1,43}$ & $\mathrm{~b}$ & $258,820.597(46)$ & 42.6 & 282.3 & & & \\
\hline $44_{0,44}-43_{0,43}$ & $\mathrm{a}$ & $258,820.597(46)$ & 43.8 & 282.3 & & & \\
\hline $43_{1,43}-42_{1,42}$ & $\mathrm{a}$ & $253,005.255(41)$ & 42.8 & 269.9 & $240_{-57}^{+68}$ & $<50$ & \\
\hline $43_{0,43}-42_{1,42}$ & $\mathrm{~b}$ & $253,005.255(41)$ & 41.6 & 269.9 & & & \\
\hline $43_{1,43}-42_{0,42}$ & $\mathrm{~b}$ & $253,005.255(41)$ & 41.6 & 269.9 & & & \\
\hline $43_{0,43}-42_{0,42}$ & $\mathrm{a}$ & $253,005.255(41)$ & 42.8 & 269.9 & & & \\
\hline $42_{1,42}-41_{1,41}$ & $\mathrm{a}$ & $247,189.500(36)$ & 41.8 & 257.7 & $273_{-57}^{+62}$ & $<100$ & \\
\hline $42_{1,42}-41_{0,41}$ & $\mathrm{~b}$ & $247,189.500(36)$ & 40.6 & 257.7 & & & \\
\hline $42_{0,42}-41_{1,41}$ & $\mathrm{~b}$ & $247,189.500(36)$ & 40.6 & 257.7 & & & \\
\hline $42_{0,42}-41_{0,41}$ & $\mathrm{a}$ & $247,189.500(36)$ & 41.8 & 257.7 & & & \\
\hline $41_{1,41}-40_{1,40}$ & $\mathrm{a}$ & $241,373.340(32)$ & 40.8 & 245.9 & $310_{-53}^{+54}$ & $<100$ & Line $27^{\mathrm{C}} \cdot$ Not masked by interlopers ${ }^{\mathrm{b}}$ \\
\hline $41_{0,41}-40_{1,40}$ & $\mathrm{~b}$ & $241,373.340(32)$ & 39.6 & 245.9 & & & \\
\hline $41_{1,41}-40_{0,40}$ & $\mathrm{~b}$ & $241,373.340(32)$ & 39.6 & 245.9 & & & \\
\hline $41_{0,41}-40_{0,40}$ & $\mathrm{a}$ & $241,373.340(32)$ & 40.8 & 245.9 & & & \\
\hline $40_{1,40}-39_{1,39}$ & $\bar{a}$ & $235,556.787(29)$ & 39.8 & 234.3 & $\cdots$ & $\cdots$ & Masked by $\mathrm{CH}_{3} \mathrm{CH}_{2} \mathrm{CN}$ at $235,562 \mathrm{MHz}^{\mathrm{b}}$ \\
\hline $40_{1,40}-39_{0,39}$ & $\mathrm{~b}$ & $235,556.787(29)$ & 38.6 & 234.3 & & & \\
\hline $40_{0,40}-39_{1,39}$ & $\mathrm{~b}$ & $235,556.787(29)$ & 38.6 & 234.3 & & & \\
\hline $40_{0,40}-39_{0,39}$ & $\mathrm{a}$ & $235,556.787(29)$ & 39.8 & 234.3 & & & \\
\hline $39_{1,39}-38_{1,38}$ & $\bar{a}$ & $229,739.850(26)$ & 38.8 & 223.0 & 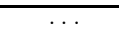 & 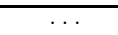 & Masked by $\mathrm{CH}_{3} \mathrm{OH}$ at $229,756 \mathrm{MHz}^{\mathrm{b}}$. \\
\hline $39_{0,39}-38_{1,38}$ & $\mathrm{~b}$ & $229,739.850(26)$ & 37.6 & 223.0 & & & \\
\hline $39_{1,39}-38_{0,38}$ & $\mathrm{~b}$ & $229,739.850(26)$ & 37.6 & 223.0 & & & \\
\hline $39_{0,39}-38_{0,38}$ & $\mathrm{a}$ & $229,739.850(26)$ & 38.8 & 223.0 & & & \\
\hline $38_{1,38}-37_{1,37}$ & $\bar{a}$ & $223,922.537(23)$ & 37.8 & 211.9 & $\cdots$ & $\cdots$ & Masked by $\mathrm{CH}_{3} \mathrm{CH}_{2} \mathrm{CN}$ at $223,932 \mathrm{MHz}^{\mathrm{b}}$ \\
\hline $38_{1,38}-37_{0,37}$ & $\mathrm{~b}$ & $223,922.537(23)$ & 36.6 & 211.9 & & & \\
\hline $38_{0,38}-37_{1,37}$ & $\mathrm{~b}$ & $223,922.537(23)$ & 36.6 & 211.9 & & & \\
\hline $38_{0,38}-37_{0,37}$ & $\mathrm{a}$ & $223,922.537(23)$ & 37.8 & 211.9 & & & \\
\hline
\end{tabular}

${ }^{\mathrm{a}} 2 \sigma$ uncertainty is in parentheses.

${ }^{\mathrm{b}}$ Nummelin et al. (1998)

${ }^{c}$ Kuan et al. (2003) list this as glycine line 27 . 
Table 5. Other Candidates for Some of the Reported Glycine Lines

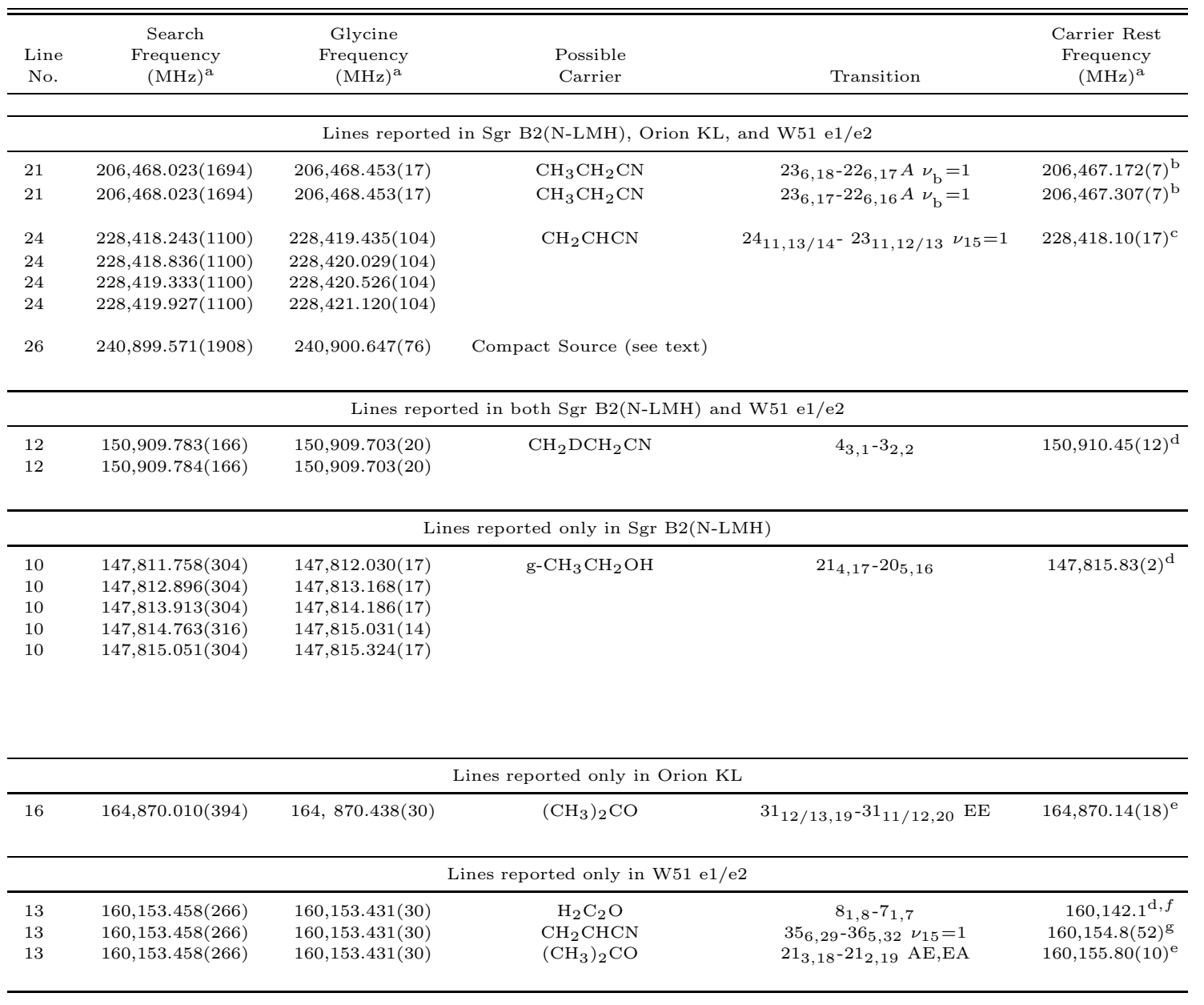

${ }^{\mathrm{a}} 2 \sigma$ uncertainty is in parentheses.

${ }^{\mathrm{b}}$ Rest frequency from J. C. Pearson, private communication (2003).

${ }^{\mathrm{c}}$ Rest frequency from Lovas (2004).

${ }^{\mathrm{d}}$ Rest frequency from Pickett et al. (1998).

${ }^{\mathrm{e}}$ Rest frequency from Groner et al. (2002).

${ }^{\mathrm{f}}$ Identified in Orion by Lee \& Cho (2002).

${ }^{\mathrm{g}}$ Rest frequency from F. J. Lovas, private communication (2004). 
Table 6. Incorrectly Assigned Glycine Transitions Used to Create the Rotational Temperature Diagram

\begin{tabular}{|c|c|c|c|c|c|c|}
\hline $\begin{array}{c}\text { Calculated }^{\mathrm{a}} \\
\text { Glycine Frequency } \\
(\mathrm{MHz}) \\
(1)\end{array}$ & $\begin{array}{l}\text { Corresponding } \\
\text { Glycine } \\
\text { Transition } \\
(2)\end{array}$ & $\begin{array}{c}S \mu^{2} \\
\left(\text { Debye }^{2}\right) \\
(3)\end{array}$ & $\begin{array}{l}E_{\mathrm{u}} \\
(\mathrm{K}) \\
(4)\end{array}$ & $\begin{array}{c}\text { Nearly Frequency } \\
\text { Coincident U Line } \\
(\mathrm{MHz}) \\
(5)\end{array}$ & $\begin{array}{c}\mathrm{U}_{\text {Line }}^{\mathrm{b}} \\
\int \Delta I d v \\
\left(\mathrm{Jy} \mathrm{beam}^{-1} \mathrm{~km} \mathrm{~s}^{-1}\right) \\
(6)\end{array}$ & $\begin{array}{c}\text { Beam } \\
\theta_{a} \times \theta_{b} \\
\left({ }^{\prime \prime} \times{ }^{\prime \prime}\right) \\
(7)\end{array}$ \\
\hline $86,210.408(367)$ & $19_{7,13}-19_{6,14}$ & 5.1 & 79.0 & U86207.8 & $7.1(16)$ & $23.9 \times 6.6$ \\
\hline $86,440.861(1000)$ & $32_{9,23}-32_{8,24}$ & 10.4 & 202.5 & U86440.2 & $9.5(28)$ & $24.1 \times 7.0$ \\
\hline $86,885.889(19)$ & $12_{3,9}-11_{3,8}$ & 9.4 & 29.4 & & $<1.9^{\mathrm{c}}$ & $25.7 \times 6.5$ \\
\hline $89,735.673(344)$ & $26_{7,20}-26_{6,21}$ & 7.4 & 132.9 & U89731.9 & $6.8(23)$ & $30.9 \times 6.3$ \\
\hline $89,831.975(10)$ & $14_{1,13}-13_{1,12}$ & 11.2 & 34.4 & U89829.6 & $5.0(17)$ & $23.8 \times 6.2$ \\
\hline $89,875.703(14)$ & $13_{5,9}-125,8$ & 9.2 & 38.3 & & $<2.4^{\mathrm{d}}$ & $24.5 \times 6.2$ \\
\hline $90,035.981(16)$ & $15_{0,15}-14_{1,14}$ & 6.6 & 35.4 & U90033.9 & $12.3(29)$ & $23.4 \times 6.6$ \\
\hline $90,043.198(16)$ & $15_{1,15}-14_{1,14}$ & 12.3 & 35.4 & $\ldots$ & $<1.8^{\mathrm{e}}$ & $23.4 \times 6.6$ \\
\hline $90,049.762(16)$ & $15_{0,15}-14_{0,14}$ & 12.3 & 35.4 & $\ldots$ & $<1.8^{\mathrm{e}}$ & $23.4 \times 6.6$ \\
\hline $90,056.979(16)$ & $15_{1,15}-14_{0,14}$ & 6.6 & 35.4 & $\ldots$ & $<2.0^{\mathrm{f}}$ & $23.8 \times 6.6$ \\
\hline $90,303.355(12)$ & $14_{2,13}-13_{1,12}$ & 4.6 & 34.4 & U90304.8 & $2.6(14)$ & $27.1 \times 6.2$ \\
\hline $106,559.495(497)$ & $24_{6,18}-23_{7,17}$ & 1.6 & 112.8 & U106559.8 & $5.6(22)$ & $19.4 \times 5.7$ \\
\hline $106,767.076(788)$ & $31_{8,24}-30_{9,21}$ & 1.7 & 186.2 & U106763.3 & $3.0(15)$ & $20.7 \times 5.3$ \\
\hline \multirow[t]{2}{*}{$109,960.322(11)$} & $16_{8,9}-15_{8,8}$ & 10.0 & 65.9 & & & \\
\hline & & & & U109959.6 & $39.1(37)$ & $22.0 \times 5.0$ \\
\hline $109,961.001(11)$ & $16_{8,8}-15_{8,7}$ & 10.0 & 65.9 & & & \\
\hline $110,089.888(121)$ & $7_{5,3}-6_{4,2}$ & 2.2 & 17.5 & U110085.6 & $28.3(49)$ & $18.9 \times 5.3$ \\
\hline $110,106.688(121)$ & $7_{5,2}-6_{4,3}$ & 2.2 & 17.5 & U110104.7 & $19.5(41)$ & $18.9 \times 5.3$ \\
\hline
\end{tabular}

${ }^{\text {a }} 2 \sigma$ frequency uncertainty in parenthesis.

${ }^{\mathrm{b}}$ Table 5 data derived from Friedel et al. (2004). Limits are $1 \sigma$; integrated intensity uncertainties are $1 \sigma$.

${ }^{\mathrm{c}} \mathrm{T}_{\text {rot }}$ diagram predicts at least a $13 \sigma$ glycine line; none detected.

${ }^{\mathrm{d}} \mathrm{T}_{\text {rot }}$ diagram predicts at least a $9 \sigma$ glycine line; none detected.

${ }^{\mathrm{e}} \mathrm{T}_{\text {rot }}$ diagram predicts at least a $18 \sigma$ glycine line; none detected.

${ }^{\mathrm{f}} \mathrm{T}_{\text {rot }}$ diagram predicts at least a $9 \sigma$ glycine line; none detected. 